\title{
Control of Coexisting Attractors with Preselection of the Survived Attractor in Multistable Chua's System: A Case Study
}

\author{
Zeric Tabekoueng Njitacke ${ }^{1},{ }^{1,2}$ Theophile Fonzin Fozin, ${ }^{3,4}$ Christian Tchito Tchapga, \\ Gervais Dolvis Leutcho, ${ }^{2,5}$ K. Marcel Wouapi, ${ }^{6}$ and Jacques Kengne ${ }^{2}$ \\ ${ }^{1}$ Department of Electrical and Electronic Engineering, College of Technology (COT), University of Buea, P.O. Box 63, \\ Buea, Cameroon \\ ${ }^{2}$ Research Unit of Automation and Applied Computer (URAIA), Electrical Engineering Department of IUT-FV, \\ University of Dschang, P.O. Box 134, Bandjoun, Cameroon \\ ${ }^{3}$ Department of Research, Development, Innovation and Training, Inchtech's, Yaoundé, Cameroon \\ ${ }^{4}$ Department of Electrical and Electronic Engineering, Faculty of Engineering and Technology (FET), University of Buea, \\ P.O. Box 63, Buea, Cameroon \\ ${ }^{5}$ Department of Physics, Faculty of Sciences, University of Mons, P.O. Box 7000, Mons, Belgium \\ ${ }^{6}$ Research Unit of Condensed Matter, Electronics and Signal Processing (UR-MACETS) Department of Physics, \\ Faculty of Sciences, University of Dschang, P.O. Box 67, Dschang, Cameroon
}

Correspondence should be addressed to Zeric Tabekoueng Njitacke; zerictabekoueng@yahoo.fr

Received 26 May 2020; Revised 23 August 2020; Accepted 30 August 2020; Published 29 September 2020

Academic Editor: Carlos-Arturo Loredo-Villalobos

Copyright (๑) 2020 Zeric Tabekoueng Njitacke et al. This is an open access article distributed under the Creative Commons Attribution License, which permits unrestricted use, distribution, and reproduction in any medium, provided the original work is properly cited.

\begin{abstract}
Although the control of multistability has already been reported, the one with preselection of the desired attractor is still uncovered in systems with more than two coexisting attractors. This work reports the control of coexisting attractors with preselection of the survived attractors in paradigmatic Chua's system with smooth cubic nonlinearity. Techniques of linear augmentation combined to system invariant parameters like equilibrium points are used to choose the desired surviving attractors among the coexisting ones. Nonlinear dynamical tools including bifurcation diagrams, standard Lyapunov exponents, phase portraits, and cross section of initial conditions are exploited to reveal the selection scenarios of the survived attractor in the multistability control process of Chua's system. The main crisis towards annihilation of multistability in Chua's system when varying the coupling strength is interior crisis and border collision. Theoretical and numerical results obtained are further validated with PSpice analysis.
\end{abstract}

\section{Introduction}

In the study of nonlinear dynamic systems, the simultaneous existence of attractors (finite or infinite), also known as multistability [1-13], extreme multistability [14-16], or megastability [17], is now in the forefront. Recall that the famous Chua's circuit is among the widely studied electronic circuits capable to display chaos [18]. When Professor Leon Chua introduced that circuit, it was intentionally built in such a way that three equilibria of the model were unstable. Based on the local stability of each point, the circuit was able to exhibit a double-scroll chaotic attractor [18]. During the investigation of this oscillator, the main challenge was to design the nonlinear part called Chua's diode. Using opamps [19], diodes [20], transistors [21], current feedback opamps [22], and inductor free CNN (cellular neural network) cells [23], many experimental results were reported on the realization of Chua's diode. Finally, by exploiting two-stage op-amp-based negative impedance converters (NICs) in parallel, a usual implementation of Chua's diode was proposed and accepted as a standard [19]. Also in [24], Bao and collaborators, during their investigation, found that, with an unstable zero saddle point and two symmetric stable nonzero node-foci, improved Chua's circuit can also generate a 
self-excited chaotic attractor. Very recently, exponential sampled-data control for T-S fuzzy systems with application to Chua's circuit has been explored [25]. Of particular interest, Chua had also presented the ability to display the coexistence of multiple attractors for the same set of system parameters but using different initial conditions [24]. This coexistence of attractors is sometimes undesirable and needs to be avoided; hence, the investigation was carried out in this work.

Remark that these coexisting solutions in a given nonlinear dynamics system can be self-excited or hidden. Recall that an attractor is called a self-excited attractor if its basin of attraction intersects with any open neighborhood of an unstable fixed point. Otherwise, it is called a hidden attractor [26]. On the contrary, hidden solutions have attraction basin which does not overlap with the neighborhood of an equilibrium point, and thus may be difficult to find numerically $[11,27-34]$. However, the localization of the latter was made possible using an algorithm proposed by Leonov et al. [35]. Such system types (with hidden attractors) can be potentially dangerous and very unpredictable (unrelated to butterfly effect). The multistability of the nonlinear system means that the system is able to exhibit different types of coexisting stable states and different forms of attraction basin for an identical set of system parameters but using different initial conditions $[2-4,8,14,36,37]$. Since the coexistence of attractors has been commonly used in image processing $[16,38]$, it becomes very urgent to control this phenomenon when, sometimes, periodic and chaotic orbits exist simultaneously. Up to date, the prominent methods reported in the relevant literature which enable to turn a multistable system to a monostable system are the noise selection [39], pseudo-forcing [12], short pulses [40], harmonic perturbation [41], intermittent feedback [42], temporal feedback [43], and linear augmentation [2, 3, 44-50]. Except for the temporal feedback and linear augmentation methods, in almost all other existing methods, the control is applied to one parameter of the system to remove on the attractors for all initial points. Thus, external control such as the temporal feedback or linear augmentation method would be preferred. Recall that, in many bistable dynamical systems, only one of the stable states is desired to track certain system performance. In this regard, Sharma et al. [47] presented control of some bistable systems with annihilation and selection of attractors using the linear augmentation scheme. This work has been carried out using two well-known paradigmatic systems that are the autonomous Chua oscillator and a neuronal system. Furthermore, the linear augmentation method has been successfully used in [46] to control the bistability property exhibited by the Lorenz-Rössler system. In 2015, the same research team [45] exploited the linear control scheme to stabilize a system to a fixed-point state even when the original system did not have any fixed point. Recently, in [43], the authors proposed the method of temporal feedback in autonomous as well as nonautonomous systems to target the coexisting attractor. The experimental realization of the introduced method was also addressed. However, all these results were based only on bistable systems.
Very recently, Fonzin Fozin et al. [2] investigated the annihilation of the coexistence of multiple stable states in a self-excited memristive hyperchaotic oscillator based on the linear augmentation method. Exploiting nonlinear analysis tools such as bifurcation diagrams, Lyapunov exponent spectrum, phase portraits, basins of attraction, and relative basin sizes, the authors show that when increasing the control parameter, the bifurcation routes followed by each of the three coexisting attractors were progressively merged in order to give a unique diagram. The results of the authors show that, for higher values of the control parameter, the multistable system with up to three coexisting attractors becomes a monostable one with only one surviving attractor. The same result was found when the same research team was addressing the control of multistability (involving three disconnected attractors) in simplified canonical Chua's oscillator with smooth hyperbolic sine nonlinearity using the linear augmentation scheme $[3,44]$. Much recently, Tabekoueng Njitacke et al. [50] investigated the coexistence of firing patterns and their control in two neurons coupled through an asymmetric electrical synapse. Their numerical results show the effectiveness of the control strategy through annihilation of the periodic coexisting firing pattern. They found that, for higher values of the coupling strength, only a chaotic firing pattern survives. From these results, it can be seen that intensive works have been done on the multistability control of the nonlinear oscillators based on the linear augmentation method. Remark that all these successful results on the control of multistability were performed so far only on systems with unique equilibrium point $[2,3,44,50]$. This unique equilibrium point excludes the possibility to target/select a desired attractor during the multistability control process. Henceforth, we propose in this work the following:

(a) To exploit the linear augmentation method to track and select one attractor among the four coexisting attractors using each of the three equilibrium points of the model

(b) To design an analog electronic circuit of controlled Chua's oscillator to further support the numerical investigation

The layout of the paper is as follows: in Section 2, we recall some basic properties of Chua's oscillator with a smooth nonlinearity. In Section 3, numerical tracking of the coexisting attractors using usual nonlinear dynamics tools is addressed. In Section 4, the linear augmentation scheme is exploited to track each of the three coexisting attractors which survive around each of the three equilibriums points. The circuit realization of the investigated model is provided in Section 5. In Section 6, some conclusions are summarized.

\section{Description of Chua's Oscillator with Smooth Nonlinearity}

Chua's oscillator with smooth cubic nonlinearity in which multistability is controlled in this work is given by dimensionless equation (1) as follows [5, 51]: 


$$
\left\{\begin{array}{l}
\frac{\mathrm{d} x}{\mathrm{~d} t}=k \alpha(y-x-f(x)), \\
\frac{\mathrm{d} y}{\mathrm{~d} t}=k(x-y+z), \\
\frac{\mathrm{d} z}{\mathrm{~d} t}=k(-\beta y-\gamma z),
\end{array}\right.
$$

where the nonlinear function $f(\cdot)$ is defined as

$$
f(x)=a x^{3}+b x .
$$

The parameter values used in this work are the ones of the model studied in [5] and are set as $\gamma=-0.75087096$, $a=-0.0375582129, b=-0.8415410391$, and $k=-1$ with $\beta$ and $\alpha$ being tunable. As it can be seen in equation (1), the model remains identical under the substitution $(x, y, z) \Leftrightarrow(-x,-y,-z)$. The stable state generated by the model will appear in symmetric pairs in $(x, y, z)$ to re-establish the real symmetry of the model. If not, the stable states produced will remain symmetric if the real symmetry of the attractors has already been re-established. This approach has been widely exploited recently to find coexisting attractors in symmetrical systems such as jerk $[6,8,52-56]$, hyperchaotic and chaotic Chua's oscillators [3, 44, 57-59], Hopfield neural networks [10, 36, 60,61], and Duffing oscillator [9], just to name a few. In addition, it is easy to show that the model processes three equilibrium points given by the following expression: $S_{0}=\left(\begin{array}{lll}0 & 0 & 0\end{array}\right)$, and $S_{1,2}=$ $\left( \pm \bar{x}_{1},( \pm \bar{x} \gamma / \gamma+\beta),((\mp \bar{x} \beta) /(\gamma+\beta))\right)$, in which $\bar{x}$ is given by $\bar{x}=\sqrt{(1 / a)((\gamma / \beta+\gamma)-1-b)}$.

\section{Selection of Coexisting Attractors: A Numerical Approach}

3.1. Computational Method. In this section, we will use traditional nonlinear analysis tools such as bifurcation diagrams, graph of maximum Lyapunov exponent, phase portraits, two-parameter diagrams, standard Lyapunov stability diagrams, and attraction basins to hunt down windows in which controlled Chua's oscillator with a smooth nonlinearity exhibits either hysteretic dynamics of parallel bifurcation branches. These various tools are computed using the Runge-Kutta formula in Turbo Pascal software with variables and constants chosen in the extended precision mode. In this contribution, we use a constant time grid of $\Delta t=0.002$, and investigations are carried out for a very long time. In this way, the transient behavior is suppressed. Some bifurcation diagrams in this work are computed either by increasing the control parameter stating from different initial conditions or using an upward and backward continuation technique. These methods are the best for finding windows in which the model displays the coexistence of bifurcations. Graph of Lyapunov exponent is calculated using the algorithm proposed by Wolf et al. [62]. Two-parameter diagrams and standard Lyapunov stability diagrams are computed by varying simultaneously two system parameters with making up of suitable colorful diagrams. These previous diagrams, as well as the basins of attraction, are obtained by numerically computing the maximum Lyapunov exponent on a grid of $350 \times 350$ values of the chosen space parameters.

\subsection{Parallel Bifurcation Branches and Coexistence of Multiple} Attractors. In the study of nonlinear dynamical systems, the coexistence of attractors for the same set of system parameters but using different initial conditions is known as multistability. Two curious and striking manifestations of such behavior are extreme or hidden extreme multistability $[63,64]$ and megastability phenomenon [17]. This phenomenon of multistability has already been found in several nonlinear systems including the memristor-based oscillator $[8,16,57]$, jerk/hyperjerk systems [55, 56, 65], and hyperchaotic Chua's oscillator [3,4], just to name a few.

Figure 1 represents a bifurcation obtained when varying the control parameter in the range $51 \leq \beta \leq 54.5$. Two sets of data with their corresponding graph of maximum Lyapunov exponent are superimposed. A large window of coexisting bifurcations which are related with parallel bifurcation branches is presented. As a result, this superposition of the bifurcations is the coexistence of multiple attractors for the same sets of system parameters. For example, when $\beta=53.6$, Chua's circuit displays the coexistence of a symmetric pair of period-4 limit cycles (black and blue) and a symmetric pair of chaotic attractors (green and red), using different initial conditions, as depicted on the three-dimensional (3D) projection of the attractors in Figure 2(a). The set of initial conditions which enable to obtain each of the previous attractors is provided in Figure 2(b).

For this same value of the control parameter, the equilibrium points of the oscillator and their stability are checked as follows: $S_{0}=\left(\begin{array}{lll}0 & 0 & 0\end{array}\right)$ with eigenvalues given by $\lambda_{1}=31.0920$ and $\lambda_{2,3}=-0.1366 \pm 7.2349 i$ and $S_{1,2}=( \pm 2.1441, \mp 0.0305, \mp 2.1746)$ with eigenvalues given by $\lambda_{1}=22.6638$ and $\lambda_{2,3}=-0.2219+7.1926 i$. Since the eigenvalues of the investigated model around the fixed points are unstable for the set of the parameter used for the study and its basin of attraction intersects with any open neighborhood of an unstable fixed point, we conclude that the coexisting attractors found are self-excited [26]. When $\beta=53.8$, Chua's circuit displays the coexistence of a symmetric pair of period-2 limit cycles (black and blue) and a symmetric pair of chaotic attractors (green and red), using different initial conditions, as depicted on the three-dimensional (3D) projection of the attractors in Figure 3(a). The basin of attraction associated to each coexisting attractor is provided in Figure 3(b). The equilibrium points of the model as well as the eigenvalues for that discrete value of the control parameter are given as $S_{0}=\left(\begin{array}{lll}0 & 0 & 0\end{array}\right)$ with eigenvalues given by $\lambda_{1}=31.0919$ and $\lambda_{2,3}=-0.1366 \pm 7.2485 i$ and $S_{1,2}=( \pm 2.1438, \mp 0.0303, \mp 2.1741)$ with eigenvalues given by $\lambda_{1}=22.6662$ and $\lambda_{2,3}=-0.2218+7.2061 i$, which further supported the unstable nature of the equilibria. From this attraction basin of Figures 2(b) and 3(b), it can be observed that each attractor has its set of initial conditions which intercepts with the one of its direct neighbor. For each 


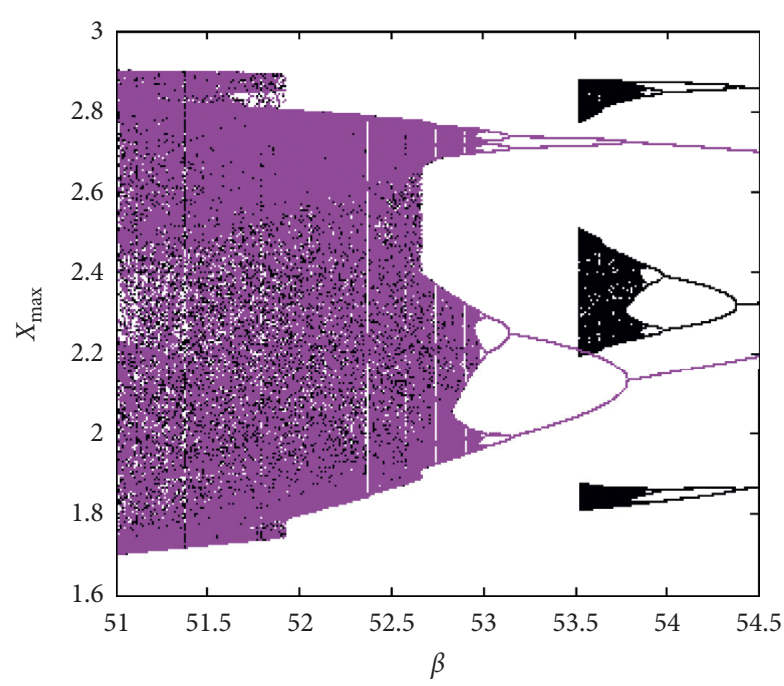

(a)

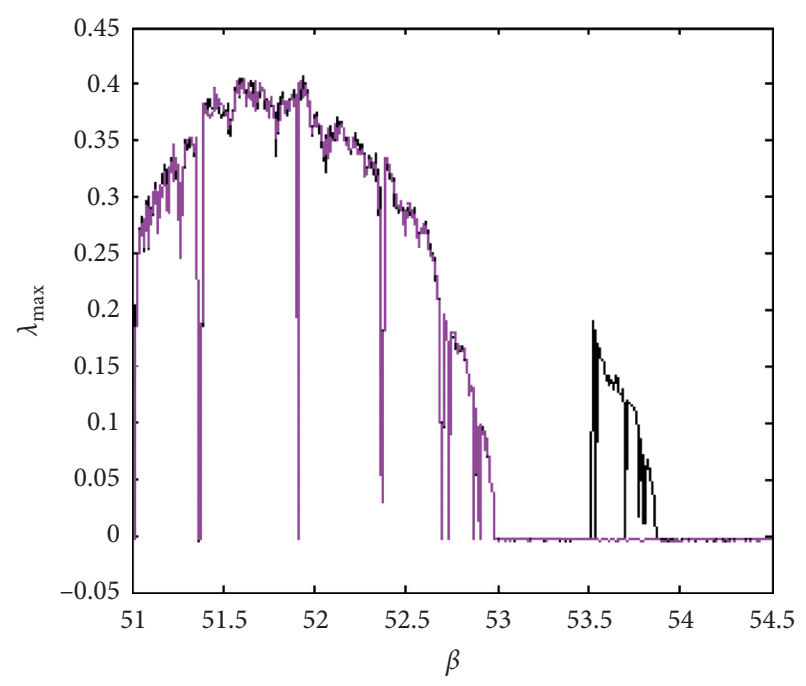

(b)

Figure 1: Bifurcation diagram (a) showing local maxima of coordinate $x$ versus $\beta$ and the corresponding graph (b) of the largest Lyapunov exponent $\left(\lambda_{\max }\right)$ plotted in the range $51 \leq \beta \leq 54.5$. Two sets of data are superimposed. The diagram in black is obtained when the control parameter is decreased from 54.5 to 51 starting with initial conditions $(1.2 ; 0 ; 0)$, while the one in magenta is obtained when decreasing the control parameter starting from the initial conditions $(1.52 ; 0 ; 0)$ with $\alpha=16.6$.

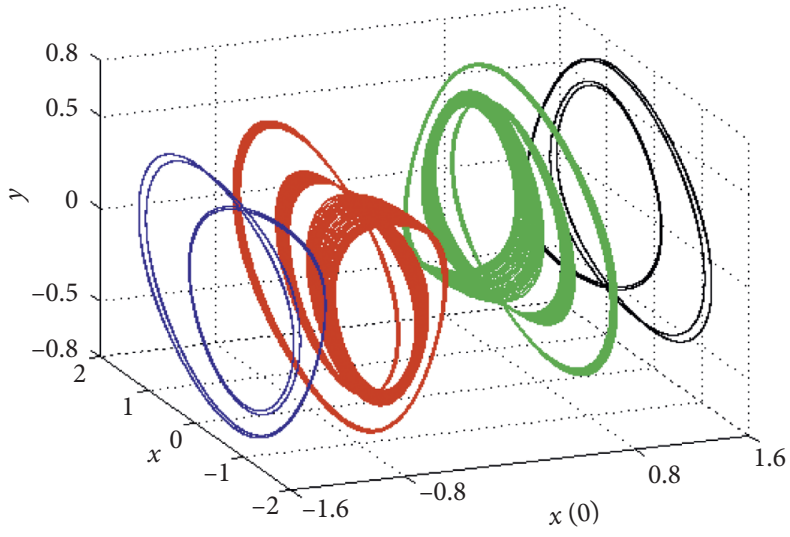

(a)

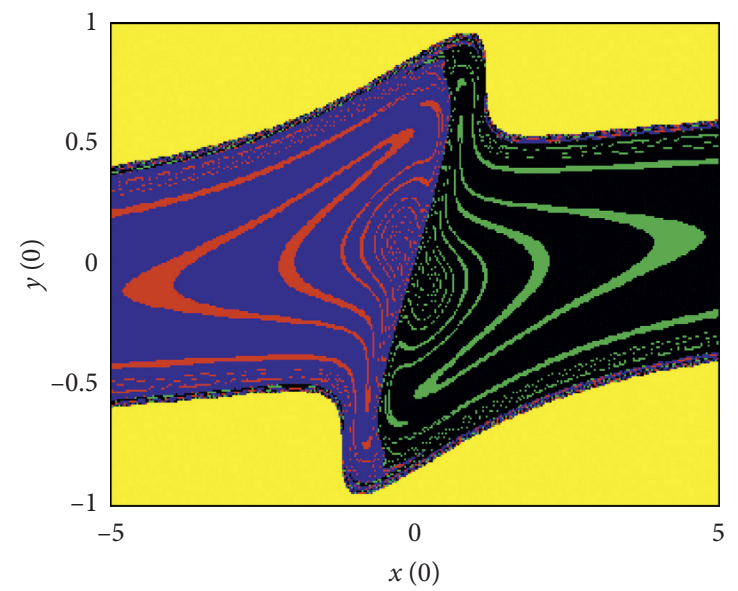

(b)

Figure 2: Three-dimensional projections of the coexisting attractors in the $(x(0), x, y)$ plane. (a) Coexistence of four different attractors (a pair of chaotic attractors and a pair of period-4 limit cycles) for $\beta=53.6$. (b) Cross sections of the basin of attraction for $z(0)=0$, corresponding to the asymmetric pair of period-4 cycles (black and blue) and the pair of chaotic attractors (red and green). Yellow regions correspond to unbounded motion.

basin of attraction, zones of unbounded motion are marked with yellow color, whereas the pair of coexisting attractors is painted in red and green, while the pair of periodic attractors is painted in blue and black.

\section{Control of Coexisting Attractors Using the Linear Augmentation Method}

4.1. Description of the Control Method. From the already published works [45-49], the theory of the linear augmentation control method consists of coupling the nonlinear system displaying multistable behavior with a linear system $(V)$ as depicted by equation (3). Remark that the choice of scalar control was guided by recent results of control and synchronization on chaotic systems [66, 67]. Indeed, it has been demonstrated that control and synchronization of the chaotic system using scalars offer great flexibility than vectors. In fact, the drawback of using vector than scalar for control or synchronization is that the full states of the systems are involved in the process. When these full states of the systems are used, the energy and resource consumption are high. 


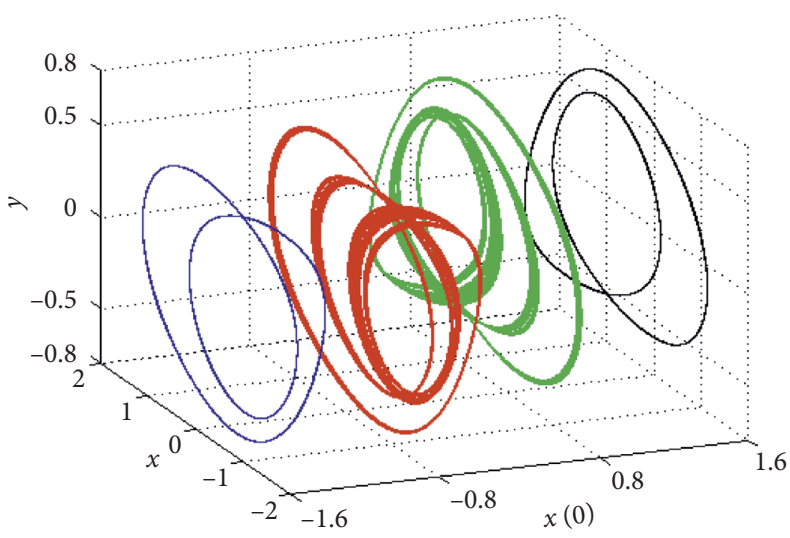

(a)

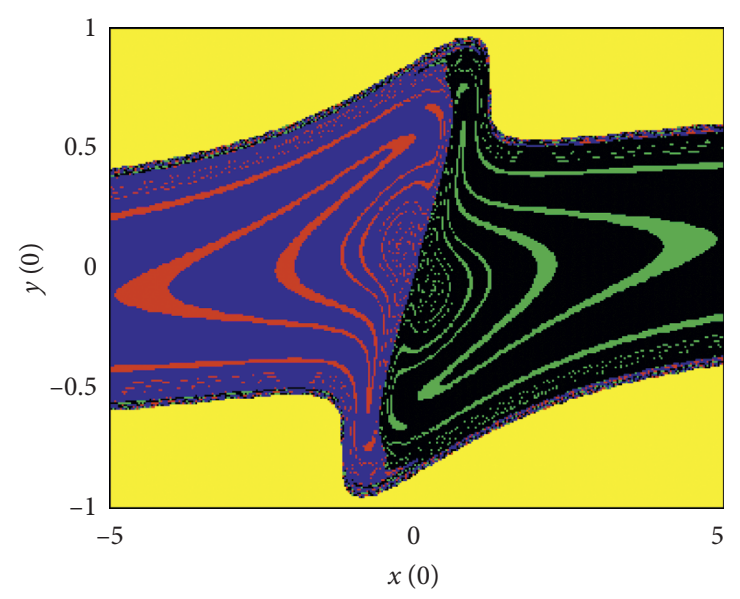

(b)

Figure 3: Three-dimensional projections of the coexisting attractors in the $(x(0), x, y)$ plane. (a) Coexistence of four different attractors (a pair of chaotic attractors and a pair of period-2 limit cycles) for $\beta=53.8$. (b) Cross sections of the basin of attraction for $z(0)=0$, corresponding to the asymmetric pair of period-2 cycles (black and blue) and the pair of chaotic attractors (red and green). Yellow regions correspond to unbounded motion.

$$
\left\{\begin{array}{l}
\dot{X}=F(X)+\delta V \\
\dot{V}=-\eta V-\delta(X-E)
\end{array}\right.
$$

In this equation, $\dot{X}=F(X)$ represents a standard form of a nonlinear dynamical system, $X$ is an $m$-dimensional vector of the system variable, and $F(X)$ is the vector field on which it is associated. Parameter $\delta$ represents the connection weight which enables to link the nonlinear system and the linear one. Vector $V$ stands for the dynamics of the linear system $\dot{V}=-\eta V$, where $\eta$ represents its decay parameter. When the controller is off, i.e., $\delta=0$, the linear system tends to zero with an exponential following a decay rate $\delta$.

$E$ represents another key parameter of controlled Chua's oscillator which will be used to track the wished stable state. It is generally selected at the neighborhood of the equilibria of the uncontrolled system [47]. These stable states, which can originate from unstable equilibria, are checked by certain sets of invariants, i.e., coexisting attractors and existing fixed points. The fixed points are found either in the center of the coexisting attractors or lying on the boundary separating the basins of attraction of the stable state. Then, by considering vector $E$ identical with one of the unstable fixed points, one can obtain the death of some of the coexisting stable states through merging crises when increasing coupling/connection weight between the coupled oscillators. For superior values of the connection weight, only one surviving attractor is obtained which enables the system for chosen parameter sets to turn from multistable to a monostable one. The controlled scheme presented above is now used for Chua's oscillator. Coupling is applied along the $x$ variable with the coupling strength $\delta$ as depicted in the following equation:

$$
\left\{\begin{array}{l}
\frac{\mathrm{d} x}{\mathrm{~d} t}=k \alpha\left(y-x-a x^{3}-b x\right)+\delta v \\
\frac{\mathrm{d} y}{\mathrm{~d} t}=k(x-y+z), \\
\frac{\mathrm{d} z}{\mathrm{~d} t}=k(-\beta y-\gamma z) \\
\frac{\mathrm{d} v}{\mathrm{~d} t}=-\eta v-\delta(x-\varepsilon) .
\end{array}\right.
$$

The equilibrium points of controlled Chua's oscillator are obtained by solving the following equation:

$$
\left\{\begin{array}{l}
k \alpha\left(y-x-a x^{3}-b x\right)+\delta v=0 \\
k(x-y+z)=0 \\
k(-\beta y-\gamma z)=0 \\
-\eta v-\delta(x-\varepsilon)=0 .
\end{array}\right.
$$

After some algebraic manipulations, we obtain the expression of the equilibrium points as follows:

$$
E_{1,2,3}=\left(\bar{x}, \frac{\bar{x} \gamma}{\gamma+\beta}, \frac{-\bar{x} \beta}{\gamma+\beta},-\frac{\delta}{\eta}(\bar{x}-\varepsilon)\right),
$$

where $\bar{x}$ is obtained by solving the following equation:

$$
\bar{x}^{3}+\frac{\bar{x}}{a}\left(1+b+\frac{\delta^{2}}{k \alpha n}-\frac{\gamma}{\gamma+\beta}\right)-\frac{\delta^{2} \varepsilon}{a k \alpha n}=0 .
$$

Considering $\quad p=(1 / a)\left(1+b+\left(\delta^{2} / k \alpha n\right)-(\gamma / \gamma+\beta)\right)$ and $q=-\left(\delta^{2} \varepsilon / a k \alpha n\right)$, equation (7) becomes

$$
\bar{x}^{3}+\bar{x} p+q=0 .
$$


The roots of (8) can be derived using the Cardano-Tartaglia method. According to the Cardano discriminant $[68,69]$, if $\Delta>0$, there is a real root and two complex roots. Since the fixed point cannot be a complex number, one equilibrium point exists.

$$
\begin{aligned}
& \bar{x}_{0}=\frac{-1+i \sqrt{3}}{2} \times \sqrt[3]{-\frac{q}{2}+\sqrt{\Delta}}+\frac{-1-i \sqrt{3}}{2} \times \sqrt[3]{-\frac{q}{2}-\sqrt{\Delta}}, \\
& \bar{x}_{1}=\sqrt[3]{-\frac{q}{2}+\sqrt{\Delta}}+\sqrt[3]{-\frac{q}{2}-\sqrt{\Delta}}, \\
& \bar{x}_{2}=\frac{-1-i \sqrt{3}}{2} \times \sqrt[3]{-\frac{q}{2}+\sqrt{\Delta}}+\frac{-1+i \sqrt{3}}{2} \times \sqrt[3]{-\frac{q}{2}-\sqrt{\Delta}},
\end{aligned}
$$

where $\Delta=(q / 2)^{2}+(p / 3)^{3}$.

If $\Delta=0$, equation (8) would have two real roots. Finally, if $\Delta<0$, there are three real roots in equation (8), which manifests that controlled Chua's oscillator has three equilibrium points and can be obtained from equations (9)-(11). The rest of the circuit parameters are the same as those used in Figure 3. The stability of controlled Chua's oscillator is provided in Table 1 . As it can be seen from Table 1, for some discrete values of the controller coefficient, controlled Chua's oscillator, under the consideration, conserves its stability, hence its ability to generate selfexcited attractors because of the unstable nature of its equilibria. It is found that when the coupling strength is null, there are three values of $\bar{x}$, among which the origin $S_{0}$ and a symmetric pair $S_{1,2}$. When the coupling strength is increased, $S_{1}$ remains uniform, while $S_{0}$ and $S_{2}$ are attracted by decreasing (resp. increasing) the value of the equilibrium points $S_{0}$ and $S_{2}$.

4.2. Control of Multistability in Chua's Oscillator. An illustrative example of the linear control method is shown in Figure 4 using the two-parameter diagram and corresponding standard Lyapunov stability diagram in the parameter space $(\delta, \beta)$. The integration method adopted here is identical with the one used in the previous section. Cyan color is tied to periodic oscillations, while magenta color is tied with chaotic motions. Good accordance is observed between two-parameter diagrams (left) and corresponding standard Lyapunov stability diagrams (right). The diagrams are obtained by sweeping upward (Figure 4(a)) and downward (Figure 4(b)) both control parameters. From a general point of view, it can be seen that, on both diagrams, four regions, namely, $(\boldsymbol{R} \mathbf{1}),(\boldsymbol{R} 2),(\boldsymbol{R} 3)$, and $(\boldsymbol{R} 4)$, can be observed. Regions ( $\mathbf{R} \mathbf{1}),(\boldsymbol{R} 2)$, and $(\boldsymbol{R} \mathbf{3})$ correspond to the set of parameters for which the model displays hysteretic dynamics which gives birth to the phenomenon of coexistence of multiple stable states, whereas $(\boldsymbol{R} 4)$ represents the set of parameters in which the model displays monostable dynamics, in other words, absence of multistability. These diagrams are very important since they enable the engineer to have a general overview on the dynamics of the multistable oscillator when the bifurcation parameter and the controller coupling strength are both varying.

Since coupling is introduced along the " $x$ " variable, we fix $\varepsilon=2.1438$ which is one among the two nontrivial equilibrium points. When increasing the control parameter $\delta$ in the range $[0 \longrightarrow 0.5]$ as it can be seen in Figure 5(a), four sets of data are superimposed in the bifurcation diagram. Each set of data (marked by red, black, blue, and green colors) corresponds to the route followed by each attractor during the control mechanism. As depicted in Figure 5(a), three crises enable all the plotted routes to merge along the one in black for higher values of the coupling strength. In region (D1) of Figure 6 and for very small values of $\delta$ (i.e., $\delta \approx 0.05$ ), four attractors coexist including two chaotic attractors (red color and green color) with two periodic attractors (black color and blue color).

At the upper boundary of (D1), the diagram in red (chaotic one) undergoes a merging crisis (first crisis) and blends with the diagram in blue. In region (D2), because of the previous merging crisis, there are only three distinct diagrams that follow their bifurcation sequences (see region (D2)). For a discrete value $\delta=0.15$, we have the coexistence of three disconnected attractors, involving a period-2, period-3, and period-4 limit cycle as presented in Figure 6(a). The demarcation region of each coexisting attractor in region (D2) is provided in Figure 6(b). As it can be observed from Figure 6(b), the basin in blue has already absorbed the one in red, while the basin in black has started to absorb the one in green. At the upper boundary of (D2), a crisis (second crisis) enables the diagram in green displaying period-3 limit cycle to merge with the diagram in black. In region $(D 3)$, we observe the superposition of two diagrams including a periodic and chaotic one.

In this region for a discrete value $\delta=0.3$, Chua's oscillator displays coexistence of a period-2 limit cycle with an asymmetric chaotic attractor (see Figure 7(a)). The basin of attraction associated with each coexisting attractor is computed and plotted in Figure 7(b). From this basin of attraction, it can be observed that the basin in black has already absorbed the one in green. At the upper boundary of (D3), a crisis (third crisis) enables the diagram in blue displaying chaotic behavior to merge with the diagram in black. In region $(D 4)$, when the critical value $\delta \approx 0.34$, all the diagrams have already merged with the black one, and the control goal is achieved as depicted in region $(D 4)$ when " $\varepsilon$ " is fixed as $\varepsilon=2.1438$. We can say that the route followed by the black diagram (see Figure $7(\mathrm{~b})$ ) is a magnetized route that attracts towards it all the other routes as the control parameter is increased.

As it can be observed in Figure 5(b), only two crises enable the control of the multistability around the origin $(\varepsilon=0)$. At the upper boundary of $(D 1)$, the first crisis enables the diagram in green to merge with the diagram in black. At the same time, the diagram in red merges with the one in blue. As presented in (D2), only a symmetrical pair of attractors coexists. At the upper boundary of (D2), the two coexisting diagrams undergo a symmetric restoring crisis 
TABLE 1: Equilibrium points of controlled Chua's oscillator, the corresponding eigenvalues, and their stability for some discrete values of the control parameter.

\begin{tabular}{lcc}
\hline Control parameter & Equilibria & Eigenvalues and stability \\
\hline \multirow{4}{*}{$\delta=0$} & $S_{0}=(0,0,0,0)$ & $31.0919,-0.1366 \pm 7.2485 i$, and -1.0 (unstable saddle focus (USF)) \\
& $S_{1}=(2.1438,-0.0303,-2.1741,0)$ & $22.6662,-0.2218 \pm 7.2061 i$, and -1.0 (unstable saddle focus (USF)) \\
& $S_{2}=(-2.1438,+0.0303,+2.1741,0)$ & $22.6662,-0.2218 \pm 7.2061 i$, and -1.0 (unstable saddle focus (USF)) \\
\hline \multirow{3}{*}{$\delta=0.2$} & $S_{1}=(2.1438,-0.0303,-2.1741,0)$ & $22.6645,-0.2218 \pm 7.2062 i$, and -0.9983 (unstable saddle focus (USF)) \\
& $S_{0}=(-0.0304,4.3 e-04,0.0308,0.4348)$ & $31.089,-0.1366 \pm 7.2485 i$, and -0.9987 (unstable saddle focus (USF)) \\
& $S_{2}=(-2.1134,0.0299,2.1433,0.8514)$ & $22.9005,-0.2188 \pm 7.2081 i$, and -0.9983 (unstable saddle focus (USF)) \\
\hline \multirow{3}{*}{0.4} & $S_{1}=(2.1438,-0.0303,-2.1741,0)$ & $22.6596,-0.2220 \pm 7.2064 i$, and -0.9930 (unstable saddle focus (USF)) \\
& $S_{0}=(-0.1273,0.0018,0.1291,0.9084)$ & $31.0571,-0.1369 \pm 7.2485 i$, and -0.9949 (unstable saddle focus (USF)) \\
& $S_{2}=(-2.0165,0.0285,2.0450,1.6641)$ & $23.6260,-0.2098 \pm 7.2136 i$, and -0.9933 (unstable saddle focus (USF)) \\
\hline
\end{tabular}
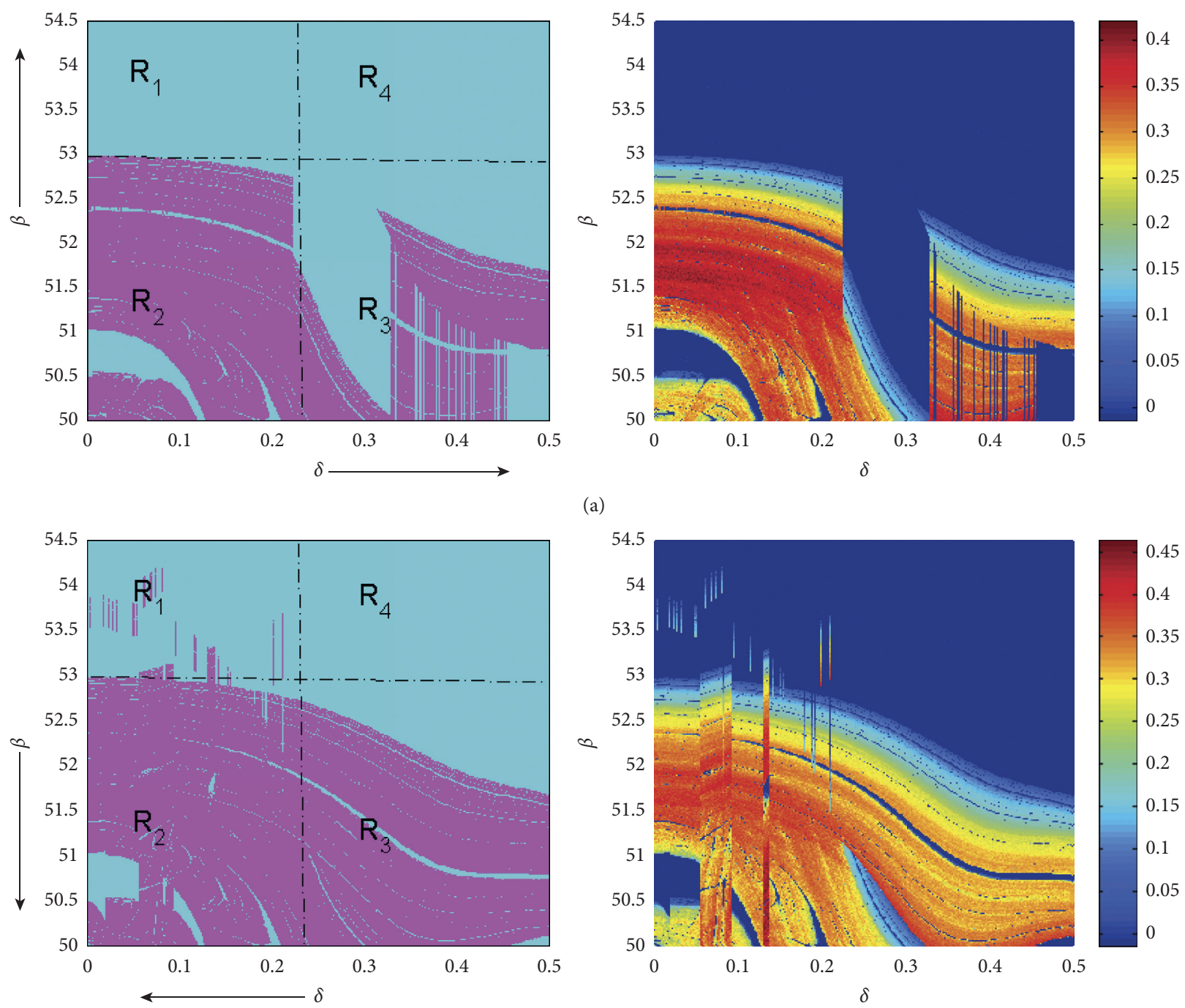

(a)

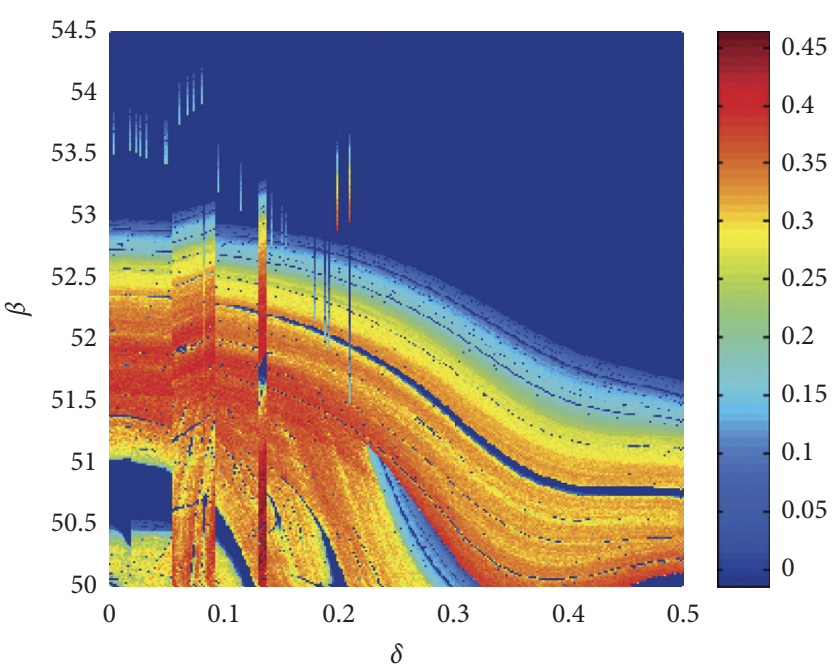

(b)

Figure 4: Two-parameter diagram (left) and the corresponding standard Lyapunov stability diagram (right) in the $(\delta, \beta)$ plane when increasing (a) and decreasing (b) both control parameters. Both diagrams show the effectiveness of the controller in the coexisting region for $\varepsilon=2.1438$. Other parameters are those of Figure 1 . 


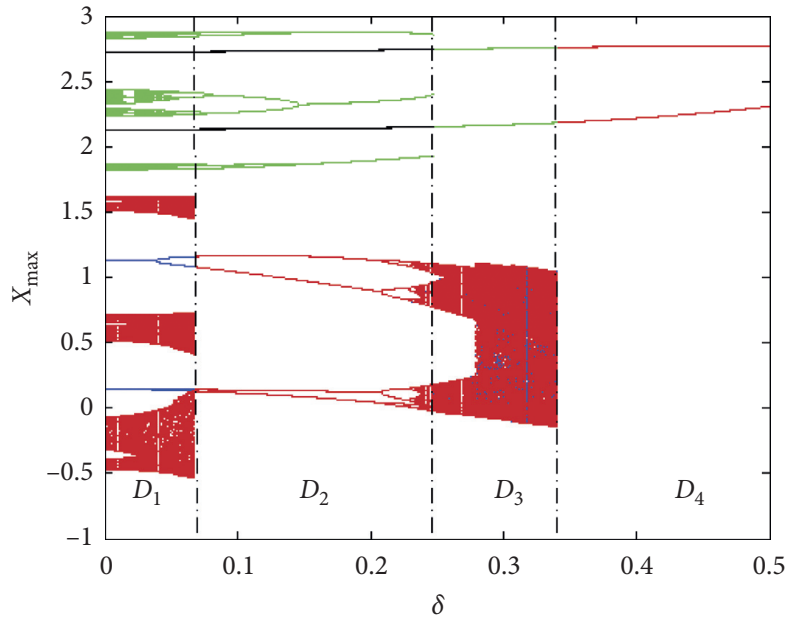

(a)

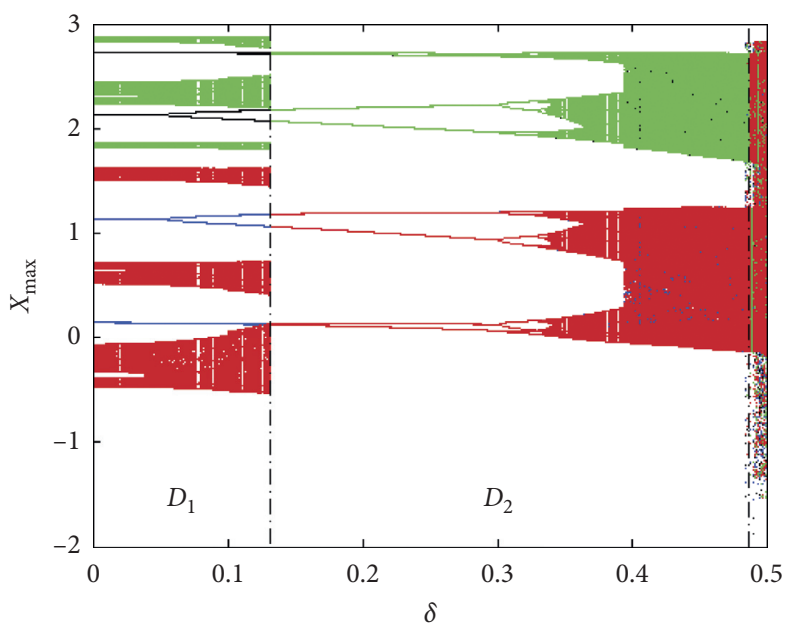

(b)

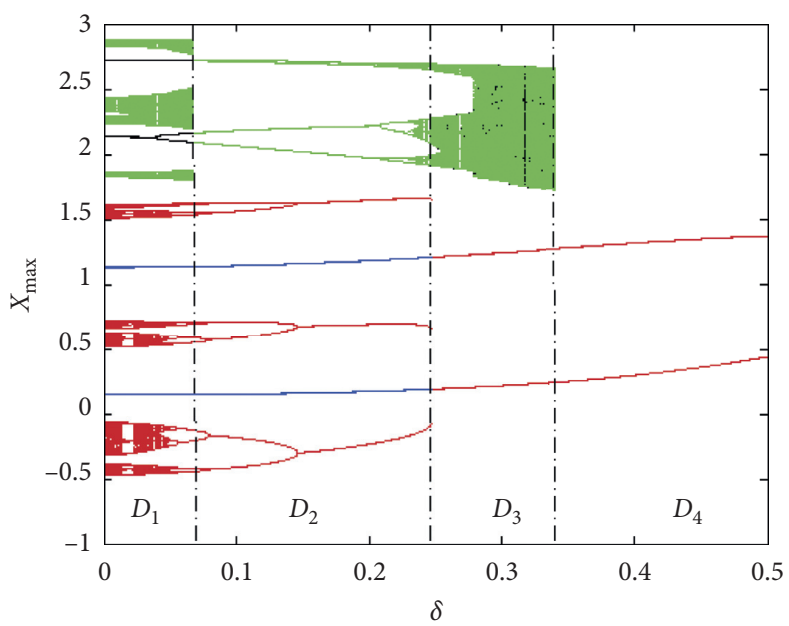

(c)

FIGURE 5: Bifurcation diagrams showing local maxima of the state variable $x$ versus the control strength $\delta$ in the range [ 00.5 ] of the controlled system (see equation (4)) showing multistability control with selection of attractors for different values of $\varepsilon$. Four separated diagrams are superimposed when increasing the coupling strength $\delta$ for four different initial conditions. Red is obtained with $(-0.56 ; 0 ; 0 ; 0)$, the one in black is obtained for $(1.6 ; 0 ; 0 ; 0)$, blue is obtained with $(-1.6 ; 0 ; 0 ; 0)$, and the one in green is obtained for $(0.56 ; 0 ; 0 ; 0)$. (a) For $\varepsilon=2.1438$, (b) for $\varepsilon=0$, and (c) for $\varepsilon=-2.1438$. For these diagrams, $\eta=1$ and $\beta=53.8$. Other parameters are those of Figure 1 .

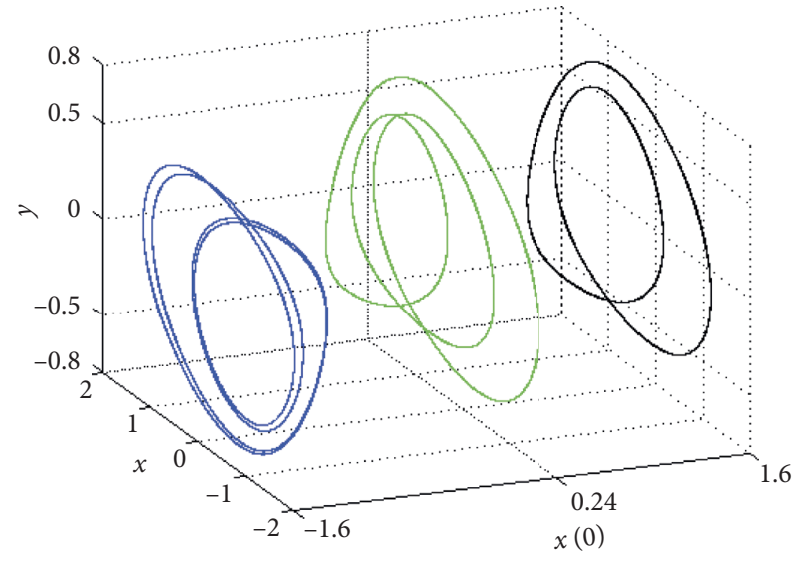

(a)

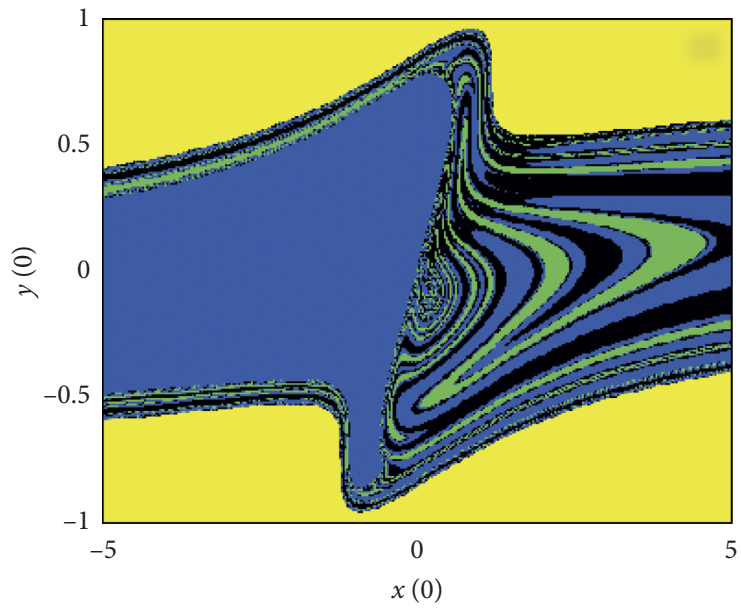

(b)

FIGURE 6: (a) Coexistence of three asymmetric periodic attractors showing multistability phenomenon with the basin of attraction (b) in the plane $(x(0), y(0))$ when $\delta=0.15$. 


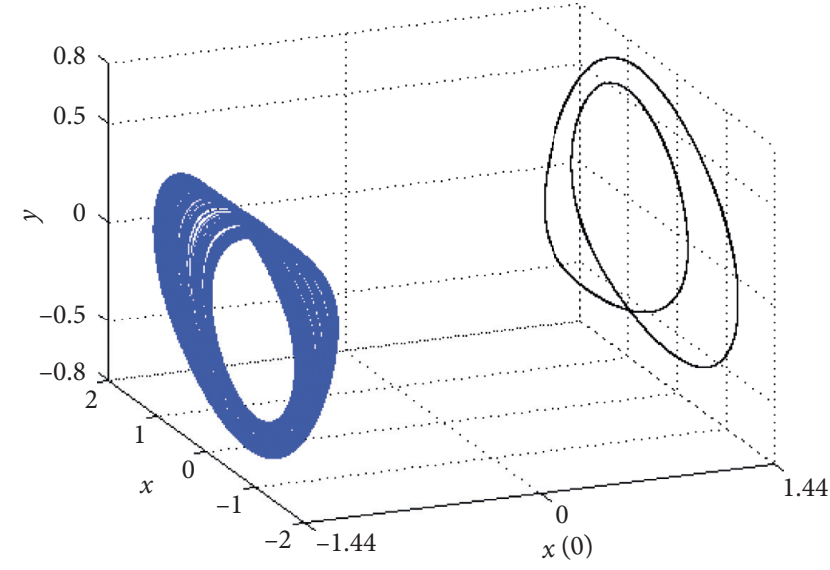

(a)

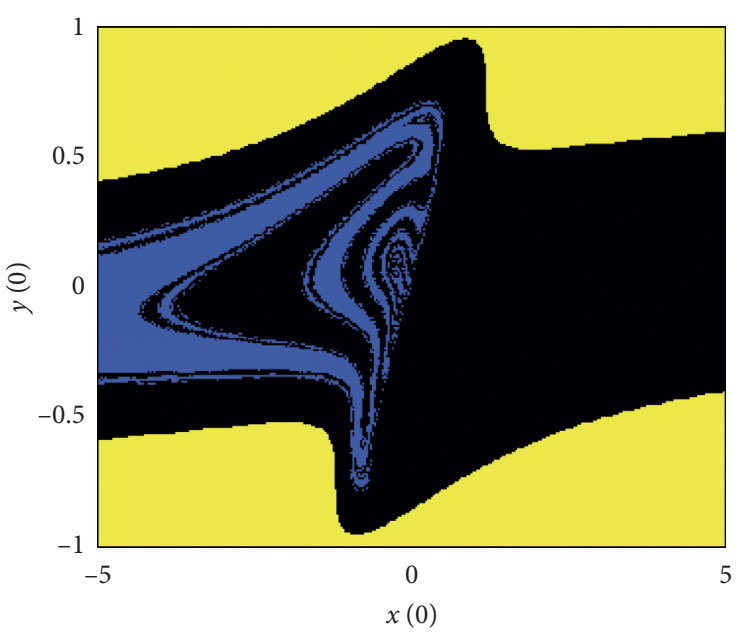

(b)

FIGURE 7: (a) Coexistence of an asymmetric chaotic attractor with a asymmetric periodic-2 limit cycle showing multistability phenomenon with the basin of attraction (b) in the plane $(x(0), y(0))$ when $\delta=0.3$.

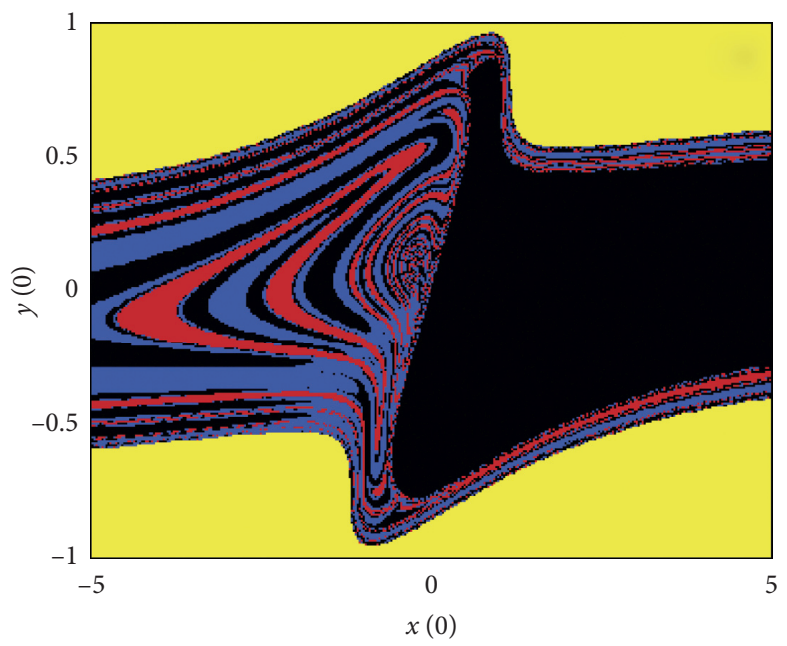

(a)

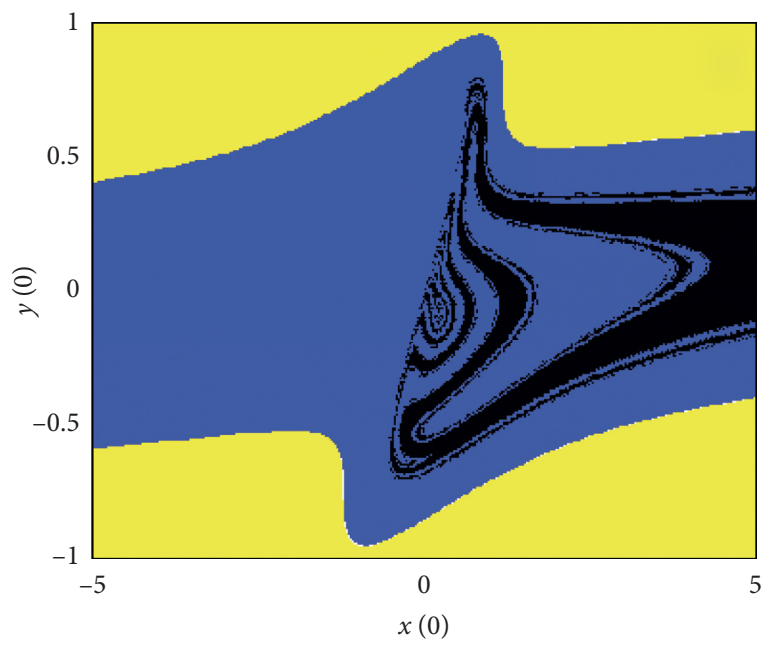

(b)

Figure 8: Basin of attractions in the plane $(x(0), y(0))$ for $\delta=0.15$ (resp. $\delta=0.3$ ) showing magnetization toward a unique stable state when the coupling strength is increased according to Figure $5(\mathrm{c})$.

and give birth to a unique diagram as depicted in region (D3).

Now, when tacking the other nontrivial equilibrium point $(\varepsilon=-2.1438)$, the surviving attractor, when monitoring the control parameter, is obtained after three crises. These crises enable all the diagrams to finally merge with the diagram in blue. The details of this control process through merging of the diagrams are not provided for the sake of brevity. However, in Figure 8, we have provided some basins of attraction to illustrate merging of the basins of attraction. In Figure 8(a), the black diagram of Figure 5(c) has already merged with the green one. For that same value, the diagram in blue starts to absorb the one in red. In Figure $8(\mathrm{~b})$, the diagram in blue has already completely merged with the red one, and only two coexisting attractors remain.

For $\delta=0.5$, we have provided the unique attractors which have survived through the control scheme exhibited by Figures 5(a)-5(c) in Figure 9(a) and their corresponding basin of attraction in Figure 9(b). It is found that when the control, the techniques described in Figure 5(a), is exploited, the attractor in black is selected. When the control, the techniques described in Figure 5(c), is used, the attractor in blue is selected, and the control method displays by Figure 5 (b) is used, the attractor in magenta is selected. These results clearly demonstrate that it is possible to target attractors based on steady points of the uncoupled system. 


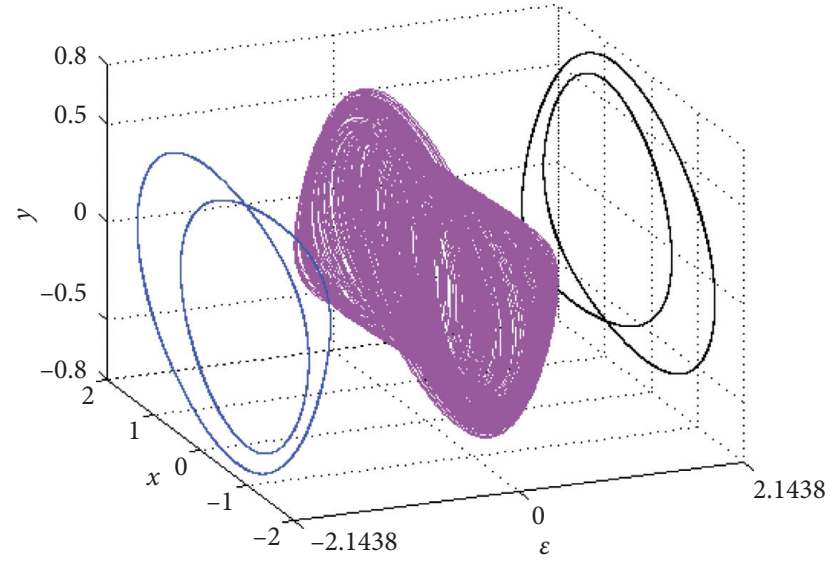

(a)

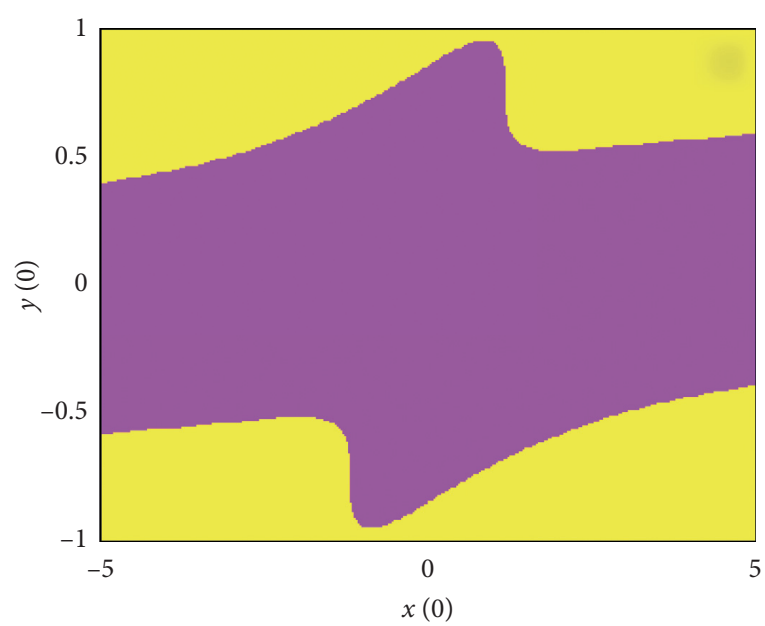

(b)

Figure 9: (a) Three-dimensional projections of the surviving stable states in the plane $(\varepsilon, x, y)$ for the typical value of the coupling $\delta=0.5$ for three distinct values of the equilibrium points, namely, $S_{0}$ and $S_{1,2}$. (b) Cross sections of the basin of attraction for $z(0)=0$, corresponding to the domain of initial conditions which enable to obtain each attractor of Figure 9(a) in magenta color. Yellow regions correspond to unbounded motion.

Also, it is worth to emphasize here that the result of the multistability control of up to four coexisting attractors in Chua's system addressed in this work has never been presented before and thus merit to be shared.

\section{The Circuit Implementation}

In this part of our work, the aim is to be able to set up an analog circuit that will allow us to make a comparison between the theoretical/numerical results obtained previously and the experimental results $[8,10,27,61,70,71]$. The circuit diagram that allows us to perform various simulations in PSpice software is presented in Figure 10. The circuit of controlled Chua's oscillator is designed using four capacitors $C_{1}, C_{2}, C_{3}$, and $C_{4}$, several resistors, eight op-amp TL082CD, a constant DC source, and two multipliers, which can be implemented practically using AD633JN versions of the AD633 four-quadrant voltage multiplier chips used to implement the nonlinear terms of our model. The signal $(W)$ at the output is related to those at inputs $X_{1}(+), X_{2}(-)$, $Y_{1}(+), Y_{2}(-)$, and $Z\left(W=\left(\left(X_{1}-X_{2}\right)\left(Y_{1}-Y_{2}\right) / 10\right)+Z\right)$. The circuit equation using Kirchhoff's electrical circuit laws can be obtained as

$$
\left\{\begin{array}{l}
C_{1} \frac{\mathrm{d} X}{\mathrm{~d} t}=\frac{1}{R_{\alpha}}\left(-Y+\frac{1}{R_{a}} X^{3}+\frac{1}{R_{b+1}} X\right)+\frac{1}{R_{\delta}} V \\
C_{2} \frac{\mathrm{d} Y}{\mathrm{~d} t}=\frac{1}{R}(-X+Y-Z), \\
C_{3} \frac{\mathrm{d} Z}{\mathrm{~d} t}=\left(\frac{1}{R_{\beta}} Y+\frac{1}{R_{\gamma}} Z\right) \\
C_{4} \frac{\mathrm{d} V}{\mathrm{~d} t}=-\frac{1}{R_{\eta}} V-\frac{1}{R_{\delta}}\left(X-V_{\varepsilon}\right) .
\end{array}\right.
$$

Setting $C_{1}=C_{2}=C_{3}=C_{4}=C=5 \mathrm{nF}, R=R_{i}=100 \mathrm{~K} \Omega$ except $R_{\alpha}, R_{b+1}, R_{\gamma}, R_{\beta}, R_{a}$, and $R_{\delta}$ and adopting the rescale of time $t=\tau R C$ and variables, $X=1 V \times x, Y=1 V \times y$, $Z=1 V \times z$, and $V=1 V \times v$, system (12) is the same with the one given in equation (4) with the following expression of parameters:

$$
\begin{aligned}
R_{\alpha} & =\frac{R}{\alpha}=6.024 \mathrm{~K} \Omega, \\
R_{\gamma} & =\frac{R}{\gamma}=133.178 \mathrm{~K} \Omega, \\
R_{\beta} & =\frac{R}{\beta}=1.85 \mathrm{~K} \Omega, \\
R_{a} & =\frac{R}{a}=2662.533 \mathrm{~K} \Omega, \\
R_{b+1} & =\frac{R}{-b+1}=631.078 \mathrm{~K} \Omega, \\
R_{\eta} & =\frac{R}{\eta}=100 \mathrm{~K} \Omega, \\
V_{\varepsilon} & =1 V \times \varepsilon, \\
R_{\delta} & =\frac{R}{\delta} .
\end{aligned}
$$

When the controller is OFF $\left(R_{\delta} \longrightarrow \infty\right.$ or $\left.\delta=0\right)$, the circuit implementation of controlled Chua's oscillator displays the phenomenon of the coexistence of up to four disconnected attractors as depicted in Figure 11. Thus, this result enables to support the fact that the previous obtained results on the coexistence of attractors in Chua's oscillator were not artifacts. When the controller is $\mathrm{ON}$ for $R_{\delta}=180 \mathrm{~K} \Omega$, selection of the attractor in controlled Chua's 

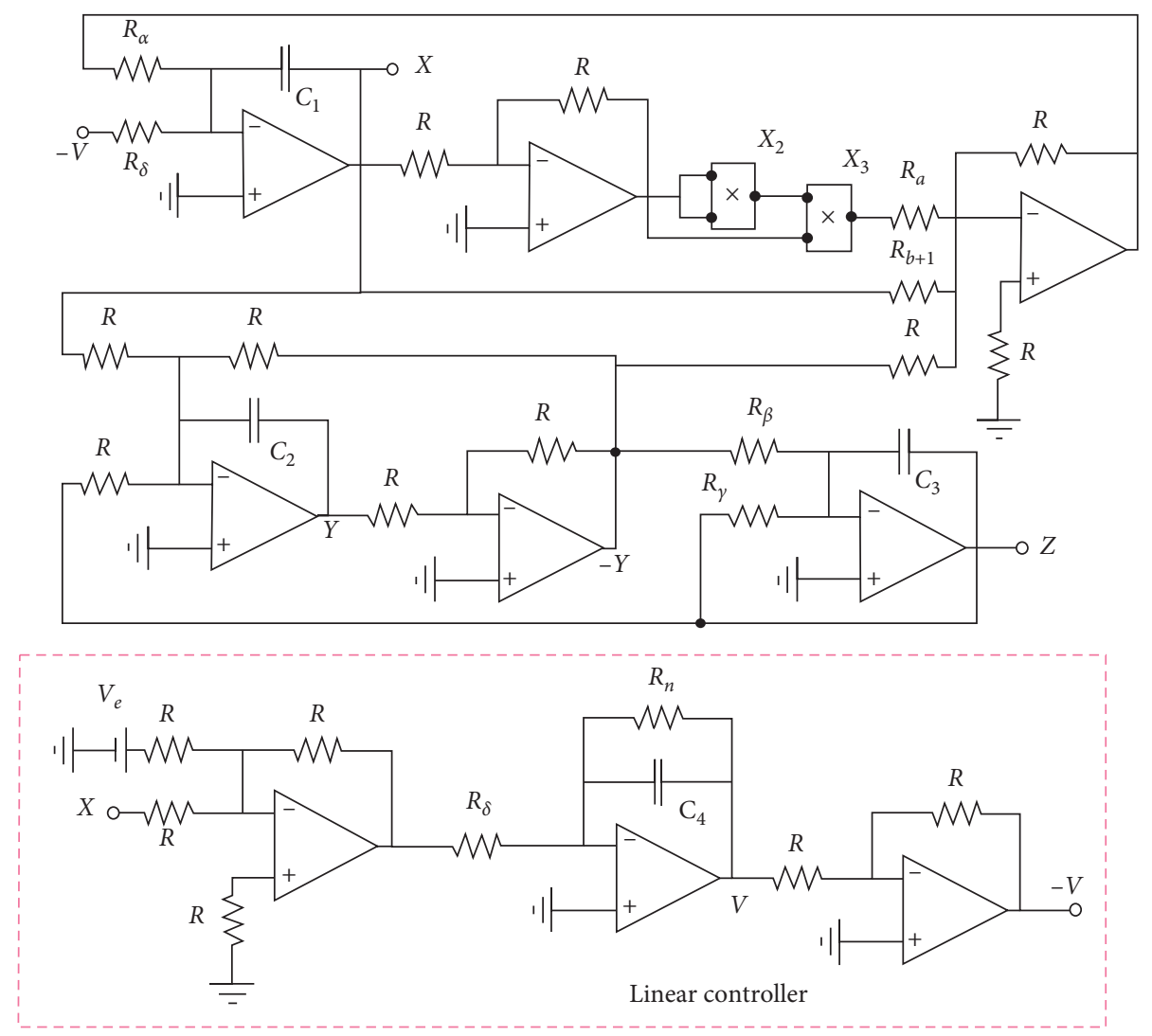

Figure 10: Schematic of Chua's oscillator coupled with the linear dynamical system.

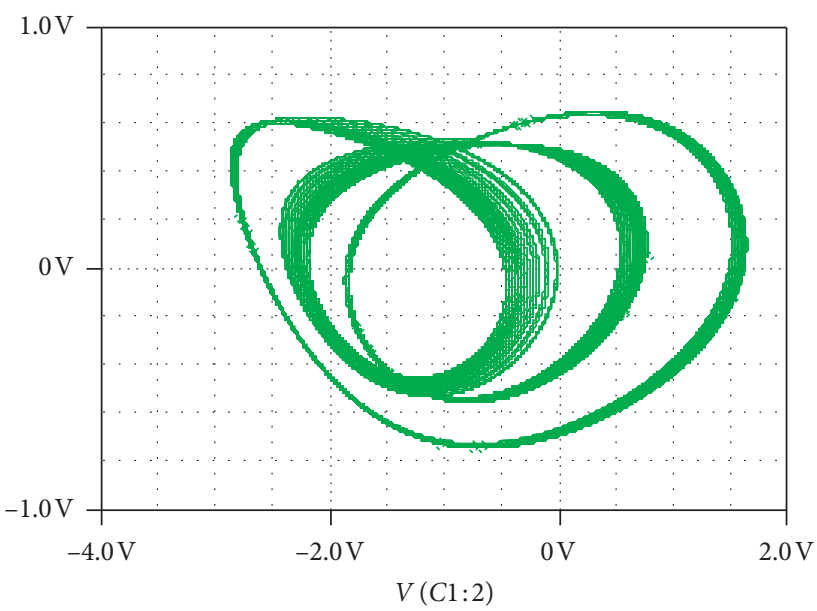

$\diamond V(C 2: 2)$

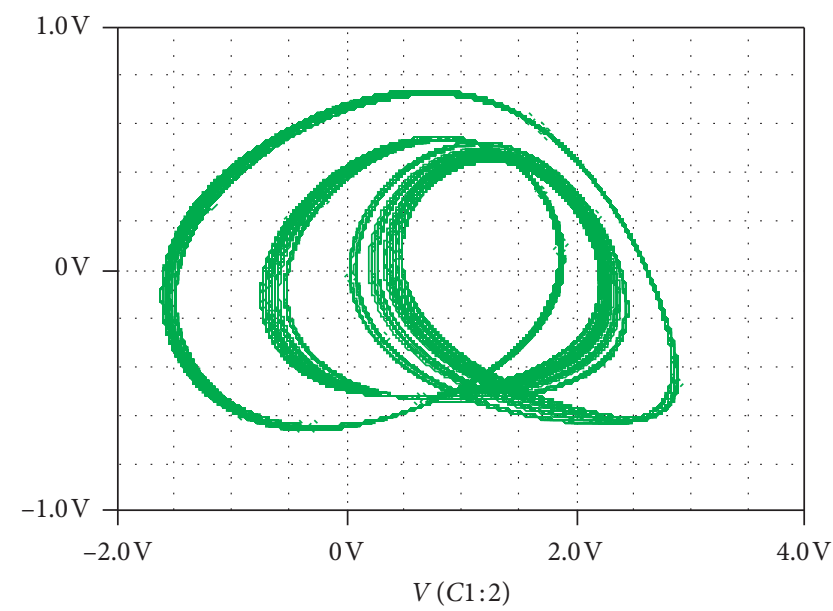

$\diamond V(C 2: 2)$

(a)

(b)

FIGURE 11: Continued. 


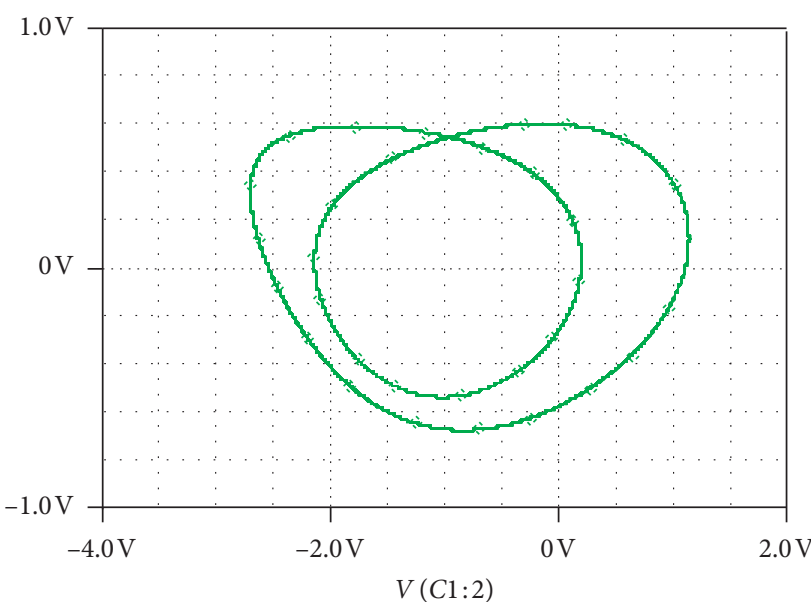

$\diamond V(C 2: 2)$

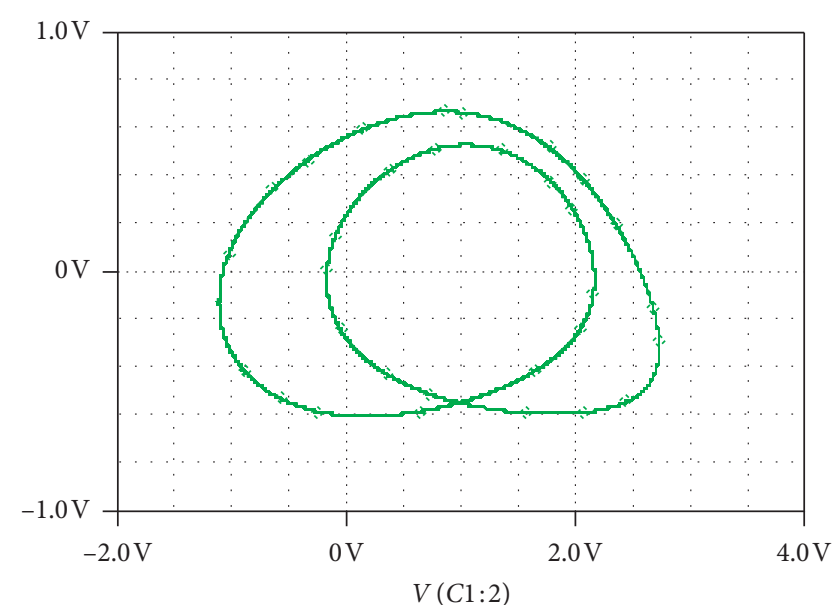

$\diamond V(C 2: 2)$

(c)

(d)

Figure 11: Phase portraits showing the coexistence of four different attractors using PSpice simulation (a pair of periodic and a pair of chaotic) for $R_{\gamma}=133.178 \mathrm{~K} \Omega$; initial conditions are $( \pm 4.3 \mathrm{~V}, \pm 0.11 \mathrm{~V}, 0 \mathrm{~V})$ for the chaotic pair and $( \pm 4.3 \mathrm{~V}, \pm 0 \mathrm{~V}, \pm 0 \mathrm{~V})$ for periodic attractors.

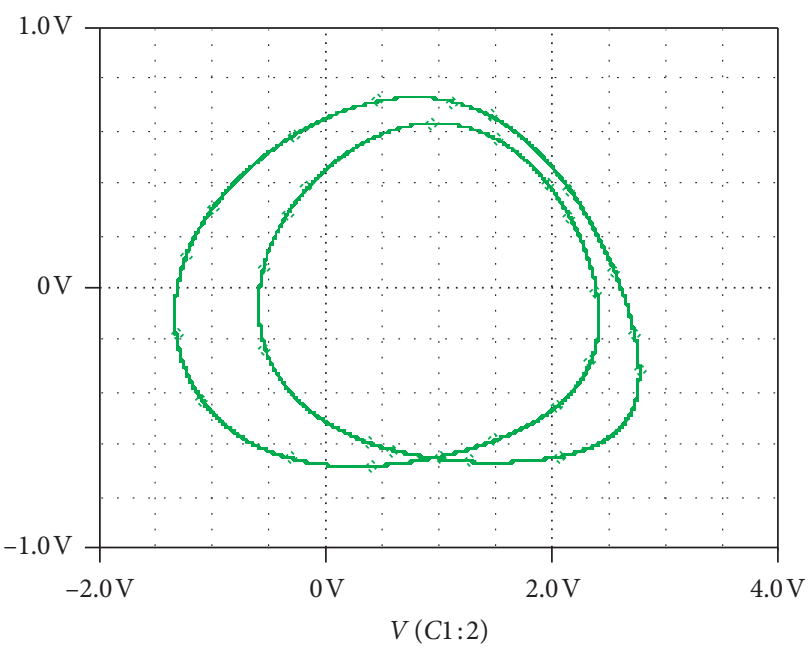

$\therefore V(C 2: 2)$

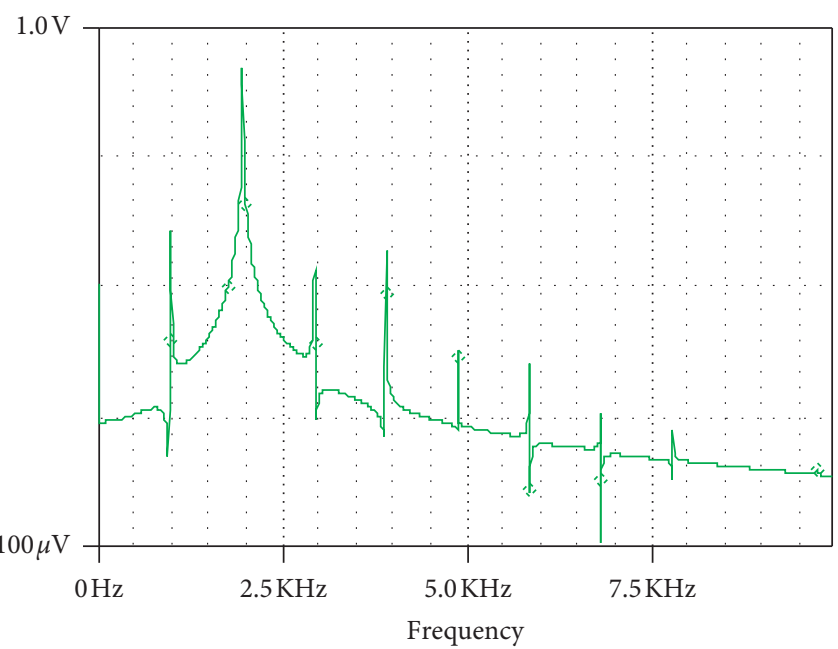

$\therefore V(C 2: 2)$

(a)

FIgURE 12: Continued. 


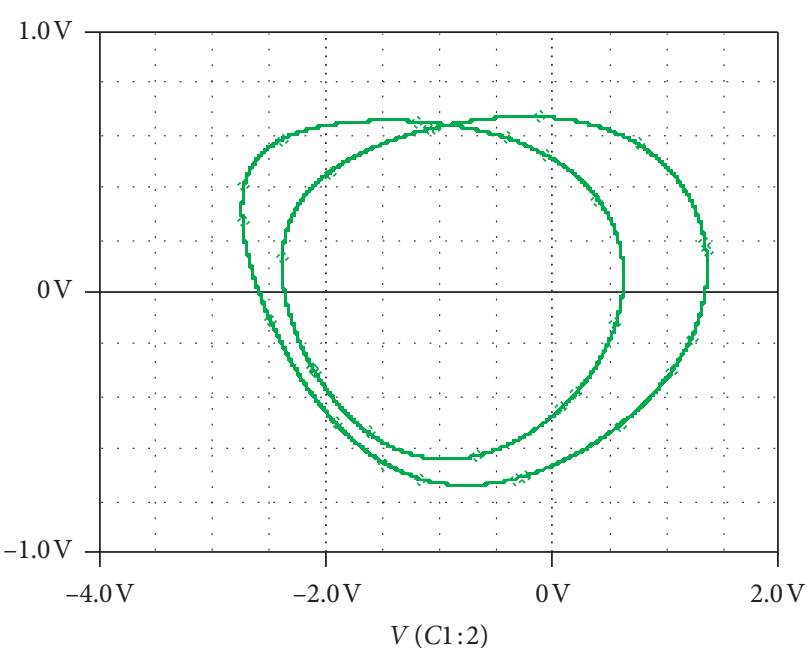

$\therefore V(C 2: 2)$

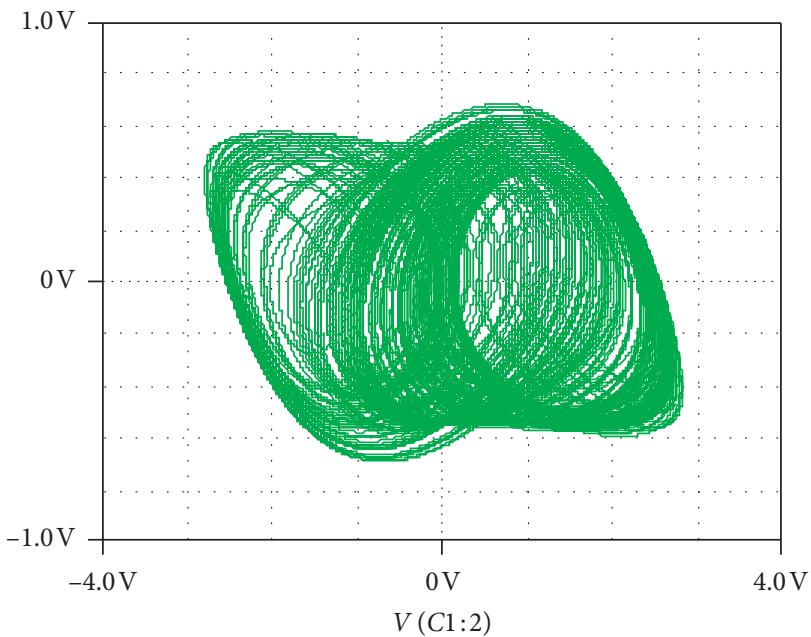

$\therefore V(C 2: 2)$

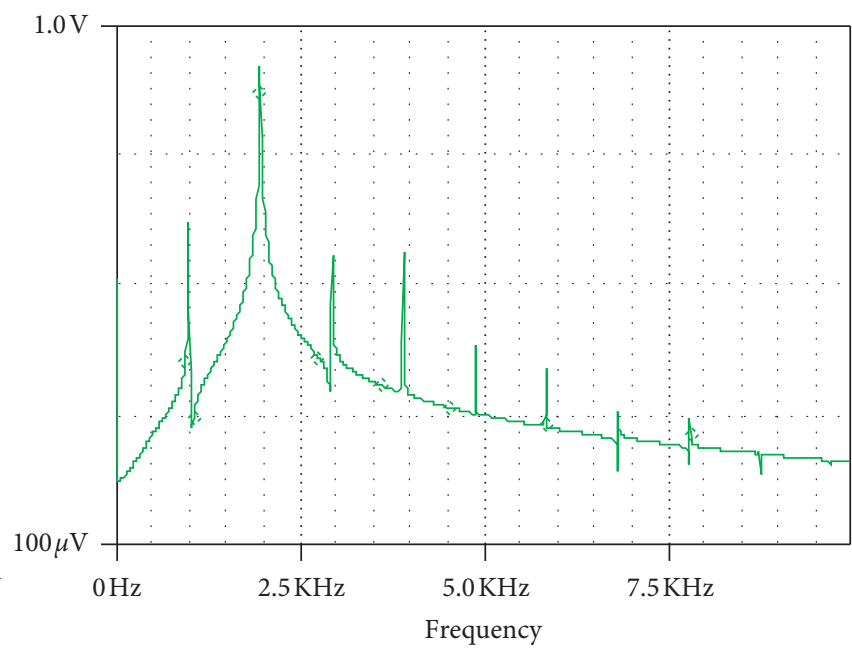

$\therefore V(C 2: 2)$

(b)

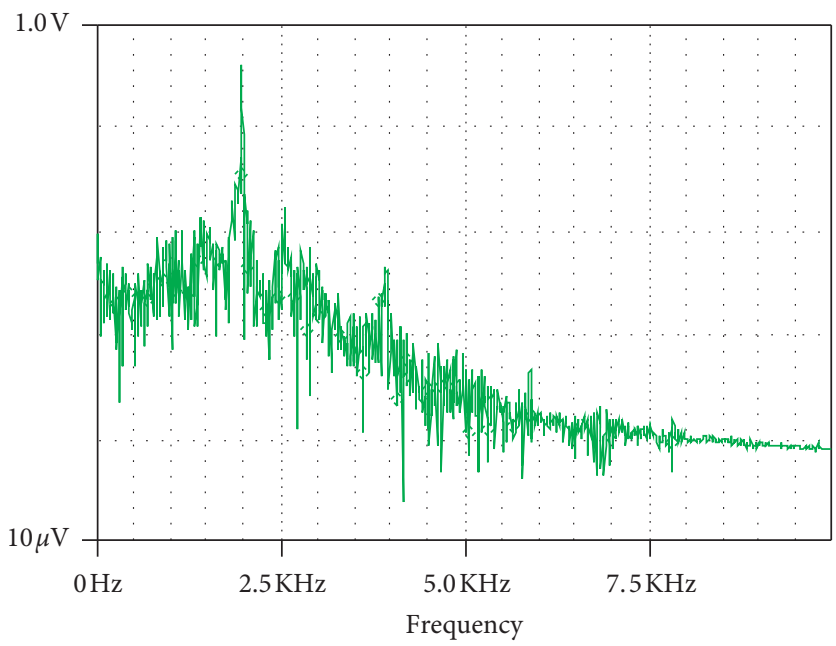

$\therefore V(C 2: 2)$

(c)

FIGURE 12: Phase portraits showing the unique surviving asymmetric and symmetric attractors for different values of the equilibrium points of the uncontrolled oscillator using PSpice simulation: (a) for $V_{\varepsilon}=2.1438 \mathrm{~V}$, (b) for $V_{\varepsilon}=-2.1438 \mathrm{~V}$, and (c) for $V_{\varepsilon}=0 \mathrm{~V}$. Parameter $R_{\delta}=180 \mathrm{~K} \Omega$, with initial conditions $(4.3 \mathrm{~V} 0 \mathrm{~V}, 0 \mathrm{~V}, 0 \mathrm{~V})$.

oscillator occurs. For example, when $R_{\delta}=180 \mathrm{~K} \Omega$ and $V_{\varepsilon}=2.1438 \mathrm{~V}$, the attractor in Figure $12(\mathrm{a})$ is selected. When $R_{\delta}=180 \mathrm{~K} \Omega$ and $V_{\varepsilon}=-2.1438 \mathrm{~V}$, the attractor in Figure $12(\mathrm{~b})$ is selected. Finally, when $R_{\delta}=180 \mathrm{~K} \Omega$ and $V_{\varepsilon}=0 \mathrm{~V}$, the pair of the symmetric attractors of Figures 12(a) and 12(b) merges and gives the double-band chaotic attractor of Figure 12(c). Besides each selected attractor, its corresponding frequency spectrum is provided to further support the nature of the attractor.

\section{Conclusion}

This paper focused on selection of the coexisting attractor in multistable Chua's oscillator with a smooth nonlinearity. The choice of paradigmatic Chua's oscillator system within this work is based on the fact that it possesses three equilibrium points and thus opens the possibility to target or select a specific coexisting attractor located around unstable equilibria. Remark that the previous studies which focused on the multistability control were done on the system having three, four, and five coexisting attractors and only one equilibrium point $[2,3,44,50]$. Based on linear augmentation, the unique equilibrium point excludes the possibility to target a coexisting attractor. This is why based on usual nonlinear techniques exploiting bifurcation diagrams, standard Lyapunov exponents, phase portraits, and crosssection basin of initial conditions, we show the possibility to control multistable Chua's oscillator (with three equilibria) towards three monostable states depending on the choice of the equilibrium point used during the linear augmentation. 
These results clearly demonstrate that it is possible to select a coexisting attractor based on steady points of the uncoupled system such as predicted by Sharma et al. [47], where only bistable systems were investigated.

\section{Data Availability}

No data were used to support this study.

\section{Conflicts of Interest}

The authors declare that they have no conflicts of interest.

\section{References}

[1] W. Ai, K. Sun, and Y. Fu, "Design of multiwing-multiscroll grid compound chaotic system and its circuit implementation," International Journal of Modern Physics C, vol. 29, no. 6, Article ID 1850049, 2018.

[2] T. Fonzin Fozin, R. Kengne, J. Kengne, K. Srinivasan, M. Souffo Tagueu, and F. B. Pelap, "Control of multistability in a self-excited memristive hyperchaotic oscillator," International Journal of Bifurcation and Chaos, vol. 29, no. 9, Article ID 1950119, 2019.

[3] T. Fonzin Fozin, P. Megavarna Ezhilarasu, Z. Njitacke Tabekoueng et al., "On the dynamics of a simplified canonical Chua's oscillator with smooth hyperbolic sine nonlinearity: hyperchaos, multistability and multistability control," Chaos: An Interdisciplinary Journal of Nonlinear Science, vol. 29, no. 11, Article ID 113105, 2019.

[4] T. F. Fonzin, K. Srinivasan, J. Kengne, and F. Pelap, "Coexisting bifurcations in a memristive hyperchaotic oscillator," AEU-international Journal of Electronics and Communications, vol. 90, pp. 110-122, 2018.

[5] J. Kengne, "On the dynamics of Chua's oscillator with a smooth cubic nonlinearity: occurrence of multiple attractors," Nonlinear Dynamics, vol. 87, no. 1, pp. 363-375, 2017.

[6] J. Kengne, R. L. T. Mogue, T. F. Fozin, and A. N. K. Telem, "Effects of symmetric and asymmetric nonlinearity on the dynamics of a novel chaotic jerk circuit: coexisting multiple attractors, period doubling reversals, crisis, and offset boosting," Chaos, Solitons \& Fractals, vol. 121, pp. 63-84, 2019.

[7] J. Kengne, Z. Njitacke Tabekoueng, V. Kamdoum Tamba, and A. Nguomkam Negou, "Periodicity, chaos, and multiple attractors in a memristor-based Shinriki's circuit," Chaos: An Interdisciplinary Journal of Nonlinear Science, vol. 25, no. 10, Article ID 103126, 2015.

[8] J. Kengne, Z. T. Njitacke, A. Nguomkam Negou, M. Fouodji Tsostop, and H. B. Fotsin, "Coexistence of multiple attractors and crisis route to chaos in a novel chaotic jerk circuit," International Journal of Bifurcation and Chaos, vol. 26, no. 5, Article ID 1650081, 2016.

[9] J. Kengne, Z. Njitacke Tabekoueng, and H. B. Fotsin, "Coexistence of multiple attractors and crisis route to chaos in autonomous third order Duffing-Holmes type chaotic oscillators," Communications in Nonlinear Science and Numerical Simulation, vol. 36, pp. 29-44, 2016.

[10] Z. T. Njitacke, J. Kengne, and H. B. Fotsin, "A plethora of behaviors in a memristor based Hopfield neural networks (HNNs)," International Journal of Dynamics and Control, vol. 7, no. 1, pp. 36-52, 2019.

[11] V.-T. Pham, C. Volos, S. Jafari, and T. Kapitaniak, "Coexistence of hidden chaotic attractors in a novel no-equilibrium system," Nonlinear Dynamics, vol. 87, no. 3, pp. 2001-2010, 2017.

[12] L. M. Pecora and T. L. Carroll, "Pseudoperiodic driving: eliminating multiple domains of attraction using chaos," Physical Review Letters, vol. 67, no. 8, pp. 945-948, 1991.

[13] Z. Huang, S. Mohamad, and H. Bin, "Multistability of HNNs with almost periodic stimuli and continuously distributed delays," International Journal of Systems Science, vol. 40, no. 6, pp. 615-625, 2009.

[14] Z. T. Njitacke, J. Kengne, R. W. Tapche, and F. B. Pelap, "Uncertain destination dynamics of a novel memristive 4D autonomous system," Chaos, Solitons \& Fractals, vol. 107, pp. 177-185, 2018.

[15] V. F. Signing and J. Kengne, "Reversal of period-doubling and extreme multistability in a novel $4 \mathrm{D}$ chaotic system with hyperbolic cosine nonlinearity," International Journal of Dynamics and Control, vol. 7, pp. 439-451, 2019.

[16] C. Li, F. Min, Q. Jin, and H. Ma, "Extreme multistability analysis of memristor-based chaotic system and its application in image decryption," AIP Advances, vol. 7, no. 12, Article ID 125204, 2017.

[17] J. C. Sprott, S. Jafari, A. J. M. Khalaf, and T. Kapitaniak, "Megastability: coexistence of a countable infinity of nested attractors in a periodically-forced oscillator with spatiallyperiodic damping," The European Physical Journal Special Topics, vol. 226, no. 9, pp. 1979-1985, 2017.

[18] L. Fortuna, Chua's Circuit Implementations: Yesterday, Today and Tomorrow, World Scientific, Singapore, 2009.

[19] M. P. Kennedy, "Robust OP Amp realization of chua's circuit,” Frequenz, vol. 46, pp. 66-80, 1992.

[20] T. Matsumoto, "A chaotic attractor from Chua's circuit," IEEE Transactions on Circuits and Systems, vol. 31, no. 12, pp. 1055-1058, 1984.

[21] T. Matsumoto, L. Chua, and K. Tokumasu, "Double scroll via a two-transistor circuit," IEEE Transactions on Circuits and Systems, vol. 33, no. 8, pp. 828-835, 1986.

[22] A. S. Elwakil and M. P. Kennedy, "Improved implementation of Chua's chaotic oscillator using current feedback op amp," IEEE Transactions on Circuits and Systems I: Fundamental Theory and Applications, vol. 47, no. 1, pp. 76-79, 2000.

[23] P. Arena, S. Baglio, L. Fortuna, and G. Manganaro, "Chua's circuit can be generated by CNN cells," IEEE Transactions on Circuits and Systems I: Fundamental Theory and Applications, vol. 42, no. 2, pp. 123-125, 1995.

[24] B. Bao, Q. Li, N. Wang, and Q. Xu, "Multistability in Chua's circuit with two stable node-foci," Chaos: An Interdisciplinary Journal of Nonlinear Science, vol. 26, Article ID 043111, 2016.

[25] N. Gunasekaran, R. Saravanakumar, M. Syed Ali, and Q. Zhu, "Exponential sampled-data control for T-S fuzzy systems: application to Chua's circuit," International Journal of Systems Science, vol. 50, no. 16, pp. 2979-2992, 2019.

[26] D. Dudkowski, S. Jafari, T. Kapitaniak, N. V. Kuznetsov, G. A. Leonov, and A. Prasad, "Hidden attractors in dynamical systems," Physics Reports, vol. 637, pp. 1-50, 2016.

[27] B. Bao, A. Hu, H. Bao, Q. Xu, M. Chen, and H. Wu, "Threedimensional memristive Hindmarsh-Rose neuron model with hidden coexisting asymmetric behaviors," Complexity, vol. 2018, Article ID 3872573, 11 pages, 2018.

[28] S. Jafari, J. C. Sprott, and S. M. R. Hashemi Golpayegani, "Elementary quadratic chaotic flows with no equilibria," Physics Letters A, vol. 377, no. 9, pp. 699-702, 2013.

[29] H. Jiang, Y. Liu, Z. Wei, and L. Zhang, "A new class of threedimensional maps with hidden chaotic dynamics," 
International Journal of Bifurcation and Chaos, vol. 26, no. 12, Article ID 1650206, 2016.

[30] V. T. Pham, S. Jafari, C. Volos, and L. Fortuna, "Simulation and experimental implementation of a line-equilibrium system without linear term," Chaos, Solitons \& Fractals, vol. 120, pp. 213-221, 2019.

[31] V. T. Pham, C. Volos, T. Kapitaniak, S. Jafari, and X. Wang, "Dynamics and circuit of a chaotic system with a curve of equilibrium points," International Journal of Electronics, vol. 105, pp. 385-397, 2018.

[32] Z. Wei, P. Yu, W. Zhang, and M. Yao, "Study of hidden attractors, multiple limit cycles from Hopf bifurcation and boundedness of motion in the generalized hyperchaotic Rabinovich system," Nonlinear Dynamics, vol. 82, no. 1-2, pp. 131-141, 2015.

[33] Z. Wei, W. Zhang, Z. Wang, and M. Yao, "Hidden attractors and dynamical behaviors in an extended Rikitake system," International Journal of Bifurcation and Chaos, vol. 25, no. 2, Article ID 1550028, 2015.

[34] Z. Wei, W. Zhang, and M. Yao, "On the periodic orbit bifurcating from one single non-hyperbolic equilibrium in a chaotic jerk system," Nonlinear Dynamics, vol. 82, no. 3, pp. 1251-1258, 2015.

[35] G. A. Leonov, N. V. Kuznetsov, and V. I. Vagaitsev, "Localization of hidden Chua's attractors," Physics Letters A, vol. 375, no. 23, pp. 2230-2233, 2011.

[36] B. Bao, H. Qian, J. Wang et al., "Numerical analyses and experimental validations of coexisting multiple attractors in Hopfield neural network," Nonlinear Dynamics, vol. 90, no. 4, pp. 2359-2369, 2017.

[37] C. Li, J. C. Sprott, W. Hu, and Y. Xu, "Infinite multistability in a self-reproducing chaotic system," International Journal of Bifurcation and Chaos, vol. 27, no. 10, Article ID 1750160, 2017.

[38] G. Peng, F. Min, and E. Wang, "Circuit implementation, synchronization of multistability, and image encryption of a four-wing memristive chaotic system," Journal of Electrical and Computer Engineering, vol. 2018, Article ID 8649294, 13 pages, 2018.

[39] A. N. Pisarchik and U. Feudel, "Control of multistability," Physics Reports, vol. 540, no. 4, pp. 167-218, 2014.

[40] V. N. Chizhevsky and S. I. Turovets, "Small signal amplification and classical squeezing near period-doubling bifurcations in a modulated $\mathrm{CO}_{2}$-laser," Optics Communications, vol. 102, no. 1-2, pp. 175-182, 1993.

[41] A. N. Pisarchik and B. K. Goswami, "Annihilation of one of the coexisting attractors in a bistable system," Physical Review Letters, vol. 84, no. 7, pp. 1423-1426, 2000.

[42] K. Yadav, N. K. Kamal, and M. D. Shrimali, "Intermittent feedback induces attractor selection," Physical Review E, vol. 95, Article ID 042215, 2017.

[43] K. Yadav, A. Prasad, and M. D. Shrimali, "Control of coexisting attractors via temporal feedback," Physics Letters A, vol. 382, no. 32, pp. 2127-2132, 2018.

[44] T. F. Fozin, G. D. Leutcho, A. T. Kouanou et al., "Multistability control of hysteresis and parallel bifurcation branches through a linear augmentation scheme," Zeitschrift für Naturforschung A, vol. 75, no. 1, pp. 11-21, 2019.

[45] P. R. Sharma, M. D. Shrimali, A. Prasad, N. V. Kuznetsov, and G. A. Leonov, "Control of multistability in hidden attractors," The European Physical Journal Special Topics, vol. 224, no. 8, pp. 1485-1491, 2015.

[46] P. R. Sharma, A. Sharma, M. D. Shrimali, and A. Prasad, "Targeting fixed-point solutions in nonlinear oscillators through linear augmentation," Physical Review E, vol. 83, Article ID 067201, 2011.

[47] P. R. Sharma, M. D. Shrimali, A. Prasad, and U. Feudel, "Controlling bistability by linear augmentation," Physics Letters A, vol. 377, no. 37, pp. 2329-2332, 2013.

[48] P. R. Sharma, M. D. Shrimali, A. Prasad, N. V. Kuznetsov, and G. A. Leonov, "Controlling dynamics of hidden attractors," International Journal of Bifurcation and Chaos, vol. 25, no. 4, Article ID 1550061, 2015.

[49] P. R. Sharma, A. Singh, A. Prasad, and M. D. Shrimali, "Controlling dynamical behavior of drive-response system through linear augmentation," The European Physical Journal Special Topics, vol. 223, no. 8, pp. 1531-1539, 2014.

[50] Z. Tabekoueng Njitacke, I. Sami Doubla, J. Kengne, and A. Cheukem, "Coexistence of firing patterns and its control in two neurons coupled through an asymmetric electrical synapse," Chaos: An Interdisciplinary Journal of Nonlinear Science, vol. 30, Article ID 023101, 2020.

[51] A. Tsuneda, "A gallery of attractors from smooth Chua's equation," International Journal of Bifurcation and Chaos, vol. 15, no. 1, pp. 1-49, 2005.

[52] J. Kengne, G. D. Leutcho, and A. N. K. Telem, "Reversals of period doubling, coexisting multiple attractors, and offset boosting in a novel memristive diode bridge-based hyperjerk circuit," Analog Integrated Circuits and Signal Processing, vol. 101, no. 3, pp. 379-399, 2019.

[53] J. Kengne and R. L. T. Mogue, "Dynamic analysis of a novel jerk system with composite tanh-cubic nonlinearity: chaos, multi-scroll, and multiple coexisting attractors," International Journal of Dynamics and Control, vol. 7, no. 1, pp. 112-133, 2019.

[54] L. K. Kengne, J. Kengne, and H. B. Fotsin, "The effects of symmetry breaking on the dynamics of a simple autonomous jerk circuit," Analog Integrated Circuits and Signal Processing, vol. 101, no. 3, pp. 489-512, 2019.

[55] G. D. Leutcho and J. Kengne, "A unique chaotic snap system with a smoothly adjustable symmetry and nonlinearity: chaos, offset-boosting, antimonotonicity, and coexisting multiple attractors," Chaos, Solitons \& Fractals, vol. 113, pp. 275-293, 2018.

[56] G. D. Leutcho, J. Kengne, and R. Kengne, "Remerging Feigenbaum trees, and multiple coexisting bifurcations in a novel hybrid diode-based hyperjerk circuit with offset boosting," International Journal of Dynamics and Control, vol. 7, no. 1, pp. 61-82, 2019.

[57] M. Chen, M. Sun, H. Bao, Y. Hu, and B. Bao, "Flux-charge analysis of two-memristor-based chua's circuit: dimensionality decreasing model for detecting extreme multistability," IEEE Transactions on Industrial Electronics, vol. 67, no. 3, pp. 2197-2206, 2019.

[58] N. Tsafack and J. Kengne, "Complex dynamics of the chua's circuit system with adjustable symmetry and nonlinearity: multistability and simple circuit realization," World Journal of Applied Physics, vol. 4, no. 2, pp. 24-34, 2019.

[59] N. Tsafack and J. Kengne, "Multiple coexisting attractors in a generalized Chua's circuit with a smoothly adjustable symmetry and nonlinearity," Journal of Physical Mathematics, vol. 10, 2019.

[60] Z. T. Njitacke, J. Kengne, and H. B. Fotsin, "Coexistence of multiple stable states and bursting oscillations in a $4 \mathrm{D}$ Hopfield neural network," Circuits, Systems, and Signal Processing, vol. 39, no. 7, pp. 3424-3444, 2020.

[61] Z. T. Njitacke and J. Kengne, "Complex dynamics of a 4D Hopfield neural networks (HNNs) with a nonlinear synaptic 
weight: coexistence of multiple attractors and remerging Feigenbaum trees," AEU-International Journal of Electronics and Communications, vol. 93, pp. 242-252, 2018.

[62] A. Wolf, J. B. Swift, H. L. Swinney, and J. A. Vastano, "Determining Lyapunov exponents from a time series," Physica D: Nonlinear Phenomena, vol. 16, no. 3, pp. 285-317, 1985.

[63] B. C. Bao, H. Bao, N. Wang, M. Chen, and Q. Xu, "Hidden extreme multistability in memristive hyperchaotic system," Chaos, Solitons \& Fractals, vol. 94, pp. 102-111, 2017.

[64] S. Zhang, Y. Zeng, Z. Li, and C. Zhou, "Hidden extreme multistability, antimonotonicity and offset boosting control in a novel fractional-order hyperchaotic system without equilibrium," International Journal of Bifurcation and Chaos, vol. 28, no. 13, Article ID 1850167, 2018.

[65] A. N. Negou and J. Kengne, "A minimal three-term chaotic flow with coexisting routes to chaos, multiple solutions, and its analog circuit realization," Analog Integrated Circuits and Signal Processing, vol. 101, pp. 415-429, 2019.

[66] K.-Y. Lian, P. Liu, T.-S. Chiang, and C.-S. Chiu, "Adaptive synchronization design for chaotic systems via a scalar driving signal," IEEE Transactions on Circuits and Systems I: Fundamental Theory and Applications, vol. 49, pp. 17-27, 2002.

[67] J. H. Peng, E. J. Ding, M. Ding, and W. Yang, "Synchronizing hyperchaos with a scalar transmitted signal," Physical Review Letters, vol. 76, no. 6, pp. 904-907, 1996.

[68] C. Han, S. Yu, and G. Wang, "A sinusoidally driven Lorenz system and circuit implementation," Mathematical Problems in Engineering, vol. 2015, Article ID 706902, 11 pages, 2015.

[69] Q. Xu, Q. Zhang, B. Bao, and Y. Hu, "Non-autonomous second-order memristive chaotic circuit," IEEE Access, vol. 5, pp. 21039-21045, 2017.

[70] Z. T. Njitacke and J. Kengne, "Nonlinear dynamics of threeneurons-based Hopfield neural networks (HNNs): remerging Feigenbaum trees, coexisting bifurcations and multiple attractors," Journal of Circuits, Systems and Computers, vol. 28, no. 7, Article ID 1950121, 2019.

[71] Q. Xu, Q. Zhang, N. Wang, H. Wu, and B. Bao, “An improved memristive diode bridge-based band pass filter chaotic circuit," Mathematical Problems in Engineering, vol. 2017, Article ID 2461964, 11 pages, 2017. 